\title{
Invited Review Article: Measurement uncertainty of linear phase-stepping algorithms
}

\author{
Erwin Hack ${ }^{1}$ and Jan Burke ${ }^{2}$ \\ ${ }^{1}$ EMPA, Laboratory Electronics/Metrology/Reliability, Ueberlandstrasse 129, CH-8600 Dübendorf, \\ Switzerland \\ ${ }^{2}$ Australian Centre for Precision Optics, CSIRO (Commonwealth Scientific and Industrial Research \\ Organisation) Materials Science and Engineering, P.O. Box 218, Lindfield, NSW 2070, Australia
}

(Received 1 February 2011; accepted 19 May 2011; published online 29 June 2011)

\begin{abstract}
Phase retrieval techniques are widely used in optics, imaging and electronics. Originating in signal theory, they were introduced to interferometry around 1970. Over the years, many robust phasestepping techniques have been developed that minimize specific experimental influence quantities such as phase step errors or higher harmonic components of the signal. However, optimizing a technique for a specific influence quantity can compromise its performance with regard to others. We present a consistent quantitative analysis of phase measurement uncertainty for the generalized linear phase stepping algorithm with nominally equal phase stepping angles thereby reviewing and generalizing several results that have been reported in literature. All influence quantities are treated on equal footing, and correlations between them are described in a consistent way. For the special case of classical $N$-bucket algorithms, we present analytical formulae that describe the combined variance as a function of the phase angle values. For the general Arctan algorithms, we derive expressions for the measurement uncertainty averaged over the full $2 \pi$-range of phase angles. We also give an upper bound for the measurement uncertainty which can be expressed as being proportional to an algorithm specific factor. Tabular compilations help the reader to quickly assess the uncertainties that are involved with his or her technique. @ 2011 American Institute of Physics. [doi:10.1063/1.3603452]
\end{abstract}

\section{INTRODUCTION}

Phase retrieval is essential for many scientific and engineering applications where periodic signals are present. These are found in methods such as interferometry, optical coherence tomography, radar, phase contrast x-ray tomography, or phased array ultrasonic testing. ${ }^{1}$ Many of the phase measurement methods rely on phase stepping whereby a series of data is acquired with defined phase changes in-between. ${ }^{2}$ In laser interferometry the periodic signal is due to the interference of coherent light beams. For phase stepping the path of one of the interfering beams is changed by, e.g., moving a mirror with a piezo actuator, tuning a liquid crystal element or stretching an optical fibre (see Fig. 1). To generate a fixed phase step, e.g., $90^{\circ}$, polarization methods can be applied. In fringe projection, the periodicity of the signal is given by the pitch of the projected fringe pattern. The fringes on the object can be realised, e.g., by an LCD projector, by projecting a glass slide with a grating, or by projecting a laser interference pattern. Phase stepping is achieved by shifting the grating in lateral direction by a fraction of the pitch. In projection moiré techniques, the reference or projected grating can be shifted. The same technique is applied in $\mathrm{x}$-ray phase contrast imaging where, e.g., the absorption grid is laterally shifted. In electronics, the periodic signal can be split in sine and cosine parts (quadrature signals) or demodulated using other homodyne techniques. In phased array ultrasonics or lock-in thermography, the periodic signal is collected and the phase is computed from a Fourier analysis. Fourier techniques can be applied to many of the other techniques as well, and are frequently used in fringe projection. Figure 2 illustrates the concept of phase stepping.

There has been much interest in designing phasestepping techniques that are insensitive to specific experimental influence quantities. ${ }^{3}$ Procedures for creating robust phase stepping algorithms include Fourier transform methods, ${ }^{4}$ linear-combination approaches, ${ }^{5}$ averaging and windowing methods, ${ }^{6-9}$ the characteristic polynomial method, ${ }^{10}$ the recursion method, ${ }^{11}$ a posteriori phase step calculations, ${ }^{12-14}$ and sophisticated statistical estimation techniques. ${ }^{15,16}$ Most of the important experimental influence quantities have been discussed individually over the years, sometimes in quite cumbersome papers. Linear phase step miscalibration arises when the phase stepping device applies a miscalibrated phase angle, demonstrated in Fig. 3. In interferometry this can be due to a mirror that is tilting, a beam that is not reflected perpendicularly to the mirror, a miscalibration of the electronic signal or hysteresis effects in the device. While an ideal interferometric signal leads to a sinusoidal variation in irradiance, higher harmonics are induced, e.g., in fringe projection when no ideal sinusoidal gratings are available.

Linear phase step miscalibration can be eliminated either by measuring the effective average phase step angle ${ }^{12-14}$ or by using an insensitive algorithm, such as the so-called five-bucket algorithm. Error minimization for linear phase step miscalibration has been specifically treated by several authors, ${ }^{17-28}$ and quadratic and cubic nonlinearities of the phase stepper have been addressed as well. ${ }^{7-9,19,29-31} \mathrm{Nu}-$ merical a posteriori correction techniques have been developed based on, e.g., iterative least-squares and so-called 


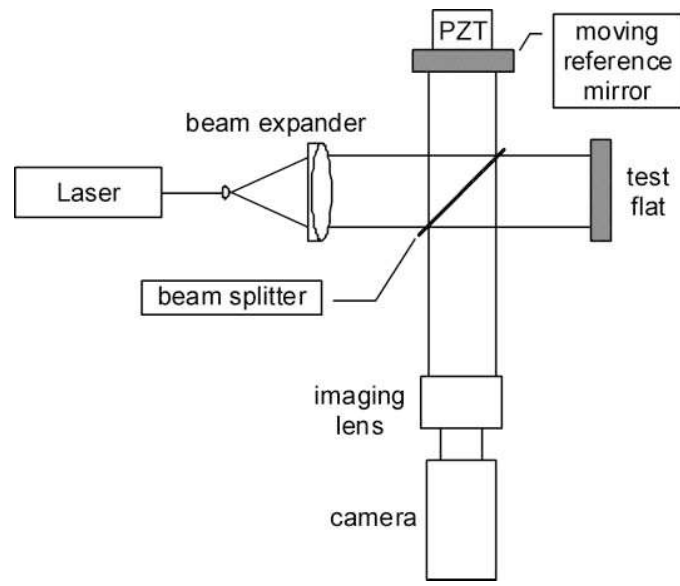

FIG. 1. Sketch of a simple phase-shifting interferometer. An expanded laser beam is divided by a beam splitter and, after reflection in each arm, recombined on a camera sensor (where the surface of the test flat should be in focus). The reference mirror is moved via a piezo-electric actuator on which it is mounted, thereby introducing the prescribed sequence of reference phase shifts.

self-calibrating methods. ${ }^{32-37}$ These techniques use the actual values of the phase step angles for reducing the measurement uncertainty. The Carré algorithm, ${ }^{12}$ like other asynchronous algorithms, ${ }^{27,38-44}$ includes an estimation of the phase step angle from the same set of measured intensities from which the phase is calculated. Consequently, such phase measurement
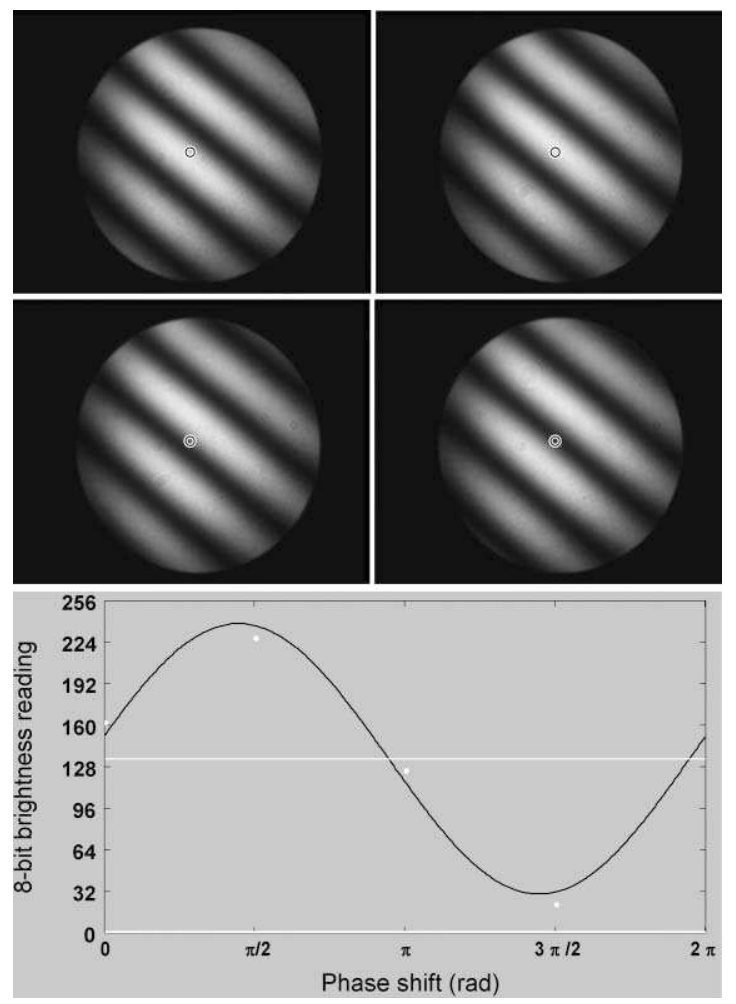

FIG. 2. Top: set of four phase-shifted fringe patterns with $90^{\circ}$ nominal phase shift per step. The pixel encircled in the centre sees a cosinusoidal brightness modulation as the reference phase is advanced. Bottom: the four brightness readings are graphed as white dots at their nominal phases; the best fit to these of a cosine is graphed in black, and the average brightness is denoted by a white line. This cosine fitting is implicitly done for every pixel in the image by application of the phase-shifting formula, and the resulting phase offset of the fitted cosine represents the desired phase $\varphi$ for each pixel.
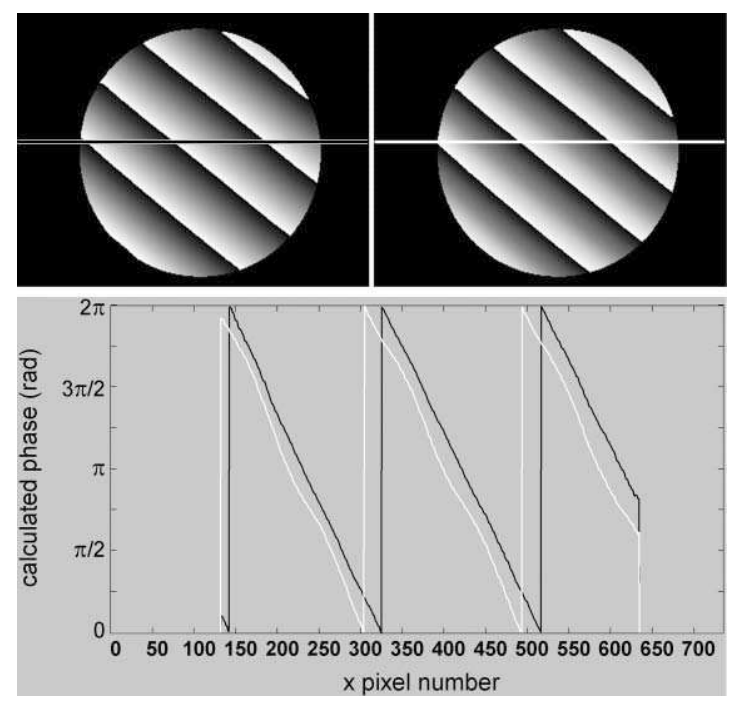

FIG. 3. Top left: "saw tooth" or "wrapped" phase map calculated from the four interferograms in Fig. 2; the phase between 0 and $2 \pi$ is represented by gray values from 0 to 255 , and the phase is usually made continuous (unwrapped) in a following data processing step. Top right: phase map from four different interferograms with $78^{\circ}$ phase shift per step, but calculated with the same $90^{\circ}$ formula as before. Bottom: plots of the central horizontal line for both saw tooth images, $90^{\circ}$ nominal and actual (black) and $90^{\circ}$ nominal and $78^{\circ}$ actual (white). The phase shows a cyclic error when the actual and nominal phase steps do not match.

algorithms are based on nonlinear signal combinations which are outside the scope of our paper.

Surrel ${ }^{10}$ has addressed the design of algorithms to eliminate higher harmonics in the signal. The minimal number of data points required to obtain insensitivity to the harmonic content of the signal up to the $j$ th order, in the presence of a linear phase-shift miscalibration, is $2 j+2$ with a phase shift of $2 \pi /(j+2)$ between frames. Phillion ${ }^{11}$ derived conditions for phase shifting algorithms to be insensitive to several influence quantities, among which are piezo distortions (resulting in wavefront tilts), background irradiance fluctuations, detector nonlinearity, and vibrations. Hibino ${ }^{20}$ showed that nonlinear phase shift, random noise, and the effect of low order harmonic components of the signal can be minimized simultaneously. $\mathrm{Zhu}^{25}$ has designed algorithms for reducing simultaneously the effects of linear phase step miscalibration and mechanical vibration.

Unfortunately, optimizing a technique for one or more influence quantities can compromise its performance with regard to others. ${ }^{20}$ In other words, once a phase stepping algorithm has been chosen, the user should be able to assess the remaining phase measurement uncertainty in view of all the relevant experimental influence quantities. Some synoptic articles are available ${ }^{45-50}$ that cite earlier quantitative results for specific algorithms from simulations. However, there are only a few comparative studies that discuss different phase shifting techniques in a quantitative manner for selected influence quantities. ${ }^{4,10,11,51-55}$ Van Wingerden et al. ${ }^{56}$ proposed a linear variational method, but it cannot be used to determine the measurement uncertainty in a consistent way. Malacara $^{26}$ and Mosiño et al. ${ }^{57}$ give a general error analysis based on a Fourier space discussion. The phase error for linear 
miscalibration is graphically quantified, but sensitivity to higher harmonics is not explicitly indicated. Hence, an approach to determine quantitatively the combined phase measurement uncertainty for a general phase stepping algorithm including most relevant influence quantities is still missing.

This paper is meant to fill the gap. We consider the uncertainty for a generalized linear phase stepping algorithm with nominally equal phase steps, and quantitatively assess - analytically and through simulations - the contribution of different influence quantities. Our approach is based on the propagation of errors ${ }^{58,59}$ rather than Monte Carlo simulations. ${ }^{53}$ The strength of the method lies in the fact that all influence quantities can be treated on equal footing, and correlations between them are described in a consistent way. An analytical model for the effect of the influence quantities is a prerequisite, but this is true also for Monte Carlo methods. Note, however, that we do not take into account influence quantities that change the phase to be measured during the procedure, e.g., speckle decorrelation.

For the general Arctan algorithms, we derive expressions for the combined variance averaged over the full $2 \pi$-range of phase angles and present an upper bound to this uncertainty. In addition, for the special case of classical $N$-bucket algorithms, we give analytic formulae that describe the dependence of the combined variance on the phase angle values. In general, the phase angle dependence of the measurement uncertainty is only of interest if a smooth phase or a specific angle value is considered, as, e.g., in a nulling interferometer. Tabular compilations will help the reader to quickly assess the uncertainties that are involved with his or her technique, either to quantify the maximum value of the influence quantity for an allowable phase measurement uncertainty, or to calculate the expected phase uncertainty for a specific set of values of the influence quantities.

\section{PHASE STEPPING ALGORITHMS OF A GENERAL ARCTAN FORM}

An important class of phase retrieval techniques is based on acquiring a series of phase stepped irradiances $I_{k}$ or signals $S_{k}$ that can be approximated by the cosinusoidal form

$$
\begin{array}{r}
I_{k}=I_{B}+I_{\Gamma} \cos \left(\varphi+\beta_{k}\right)=I_{B}\left(1+v \cos \left(\varphi+\beta_{k}\right)\right), \\
S_{k}=\gamma I_{k}=S_{B}+S_{\Gamma} \cos \left(\varphi+\beta_{k}\right),
\end{array}
$$

where $I_{B}$ represents the background irradiance or DC bias, $I_{\Gamma}$ the modulation irradiance. In imaging applications, their ratio $v$ is called fringe visibility or fringe contrast. An ideal detector converts the irradiance linearly into an electrical signal, where $\gamma$ is the detector gain. The index $k=0, \ldots, N-1$ enumerates the $N$ phase-stepped quantities, and $\beta_{k}$ is the corresponding phase step angle. The phase $\varphi$ can be retrieved from a set of arbitrarily phase stepped signals $S_{k}$ by using a linear least squares or maximum likelihood algorithm. ${ }^{47,60-64}$ Phase retrieval by the Fourier transform approach is equivalent to this least squares approach, and a choice of equal sampling intervals is least sensitive to (uncorrelated) noise. ${ }^{65}$ Since we restrict our analysis to linear combinations of the signals $S_{k}$ to synthesize sine and cosine modulation signals, we can formulate the general expression

$$
\varphi+\alpha=\arctan \left(\frac{\sum_{k=0}^{N-1} b_{k} S_{k}}{\sum_{k=0}^{N-1} a_{k} S_{k}}\right)=\arctan \left(\frac{G \sin (\varphi+\alpha)}{G \cos (\varphi+\alpha)}\right),
$$

where the real coefficients $a_{k}$ and $b_{k}$ define the implemented algorithm; these are often scaled to become integers, so that we include a real scaling factor $G$ for completeness. Arctan is short for the Arctan 2 function defined over the full $2 \pi$ range of phase values. The offset angle $\alpha$ is introduced to allow for a shift in the origin of the phase stepping angles. The following vector and complex notations will simplify the discussion: ${ }^{8,10}$

$$
\begin{gathered}
\mathbf{a}=\left(\begin{array}{c}
a_{0} \\
a_{1} \\
\vdots \\
a_{N-1}
\end{array}\right) \mathbf{b}=\left(\begin{array}{c}
b_{0} \\
b_{1} \\
\vdots \\
b_{N-1}
\end{array}\right) \mathbf{S}=\left(\begin{array}{c}
S_{0} \\
S_{1} \\
\vdots \\
S_{N-1}
\end{array}\right) \\
\mathbf{A}=\left(\begin{array}{c}
\cos \left(\beta_{0}\right) \\
\cos \left(\beta_{1}\right) \\
\vdots \\
\cos \left(\beta_{N-1}\right)
\end{array}\right) \mathbf{B}=\left(\begin{array}{c}
\sin \left(\beta_{0}\right) \\
\sin \left(\beta_{1}\right) \\
\vdots \\
\sin \left(\beta_{N-1}\right)
\end{array}\right), \\
c_{k}=a_{k}+i b_{k}=\left|c_{k}\right| \exp \left(i \delta_{k}\right), \\
C_{k}=A_{k}+i B_{k}=\exp \left(i \beta_{k}\right), \\
G \exp (i(\varphi+\alpha))=\sum_{k=0}^{N-1} c_{k} S_{k}=\mathbf{a} \cdot \mathbf{S}+i(\mathbf{b} \cdot \mathbf{S}) .
\end{gathered}
$$

Note that the coefficients are not necessarily orthogonal in the sense $\mathbf{a} \cdot \mathbf{b} \neq 0$, where the dot operation denotes the scalar product on $\mathbb{C}^{\mathrm{N}}$, i.e., $\mathbf{a} \cdot \mathbf{b}=\sum_{k} a_{k} b_{k}^{*}$. By construction, $\left|C_{k}\right|=1, \mathbf{C} \cdot \mathbf{C}=N$.

Introducing the real valued signal, Eq. (1) into Eq. (4)

$$
\begin{aligned}
G \exp (i(\varphi+\alpha))= & S_{B} \sum_{k=0}^{N-1} c_{k}+S_{\Gamma} \sum_{k=0}^{N-1} c_{k} \frac{1}{2} \\
& \times\left[\exp \left(i\left(\varphi+\beta_{k}\right)\right)+\exp \left(-i\left(\varphi+\beta_{k}\right)\right)\right],
\end{aligned}
$$

results in the following constraints on the coefficients:

(i) In order to eliminate the background signal, $S_{B}$, the sum of the coefficients must vanish ${ }^{11}$

$$
\sum_{k=0}^{N-1} c_{k}=0 .
$$

(ii) The modulus $G$ must be independent of $\varphi$, i.e., the term proportional to $\exp (-i \varphi)$ must vanish 


$$
\begin{aligned}
& 0=\exp (-i \varphi) \sum_{k=0}^{N-1} c_{k}\left(\exp \left(-i \beta_{k}\right)\right), \\
& 0=(\mathbf{a} \cdot \mathbf{A}+\mathbf{b} \cdot \mathbf{B})+i(\mathbf{b} \cdot \mathbf{A}-\mathbf{a} \cdot \mathbf{B}),
\end{aligned}
$$

from which we find

$$
\mathbf{c} \cdot \mathbf{C}=0 \text {, i.e., } \mathbf{a} \cdot \mathbf{B}=\mathbf{b} \cdot \mathbf{A}, \mathbf{a} \cdot \mathbf{A}=-\mathbf{b} \cdot \mathbf{B} .
$$

This links the coefficients $\mathbf{c}$ to the phase step angles via C, and $G$ is calculated from Eq. (4) by

$$
G^{2}=\frac{1}{4} S_{\Gamma}^{2}\left|\mathbf{c} \cdot \mathbf{C}^{*}\right|^{2}
$$

Thus, the modulus $G$ is proportional to the modulation signal $S_{\Gamma}$ and, using the Cauchy-Schwarz-Bunjakowski inequality, we have

$$
G^{2} \leq \frac{1}{4} S_{\Gamma}^{2}|\mathbf{c}|^{2}|\mathbf{C}|^{2}=S_{\Gamma}^{2}|\mathbf{c}|^{2} \frac{N}{4} .
$$

Any rotation c' of a valid algorithm $\mathbf{c}$ based on the same set of signals $\left\{S_{k} \mid k=1, \ldots, N\right\}$ yields another valid algorithm, i.e.,

$$
\begin{gathered}
\mathbf{c}^{\prime}=\mathbf{c} \exp (i \gamma), \\
a_{k}^{\prime}=a_{k} \cos \gamma-b_{k} \sin \gamma, \\
b_{k}^{\prime}=b_{k} \cos \gamma+a_{k} \sin \gamma,
\end{gathered}
$$

because the conditions Eqs. (6) and (7) are rotation invariant. The only difference is that instead of phase $\varphi$ the angle $(\varphi+$ $\gamma$ ) is measured. Most notably, the following replacements are frequently found,

$$
\begin{aligned}
& \mathbf{a} \rightarrow-\mathbf{b} \\
& \mathbf{b} \rightarrow \mathbf{a}
\end{aligned} \text { for } \gamma=90^{\circ}, \quad \text { and } \quad \begin{aligned}
& \mathbf{a} \rightarrow \mathbf{a}-\mathbf{b} \\
& \mathbf{b} \rightarrow \mathbf{a}+\mathbf{b}
\end{aligned} \text { for } \gamma=45^{\circ} .
$$

Any linear combination of valid algorithms based on the same set of signals $\left\{S_{k} \mid k=0, \ldots, N-1\right\}$, i.e., $\mathbf{c}=\lambda \mathbf{c}_{1}+$ $\mu \mathbf{c}_{2}$, yields a new valid algorithm, ${ }^{5}$ because from Eq. (4)

$$
\begin{aligned}
\left(\lambda \mathbf{c}_{1}+\mu \mathbf{c}_{2}\right) \cdot \mathbf{S}= & \lambda G_{1} \exp \left(i\left(\varphi+\alpha_{1}\right)\right) \\
& +\mu G_{2} \exp \left(i\left(\varphi+\alpha_{2}\right)\right), \\
= & \exp (i \varphi)\left[\lambda G_{1} \exp \left(i \alpha_{1}\right)+\mu G_{2} \exp \left(i \alpha_{2}\right)\right] \\
= & G \exp (i(\varphi+\alpha)) .
\end{aligned}
$$

Equation (13) is at the core of averaging algorithms, $3,6,7,17-19,26,30,31,66$ including the so-called $N+M$ algorithms, where more than one period of the signal is captured. Most frequently, $N+1$ algorithms are used to suppress the effects of linear phase step miscalibration, while the $N+(N-2)$ algorithm can be made insensitive to linear miscalibration and harmonics up to order $j=N-2 .^{10}$ Although the additional frames are nominally identical with the corresponding frames of the first period, they compensate some of the systematic uncertainty contributions.

Note that Eqs. (2)-(13) are valid for arbitrary values of the phase stepping angles. However, most algorithms build on nominally equal phase step increments $\beta$, i.e., the individual phase step angle is written as

$$
\beta_{k}=\left(k-k_{0}\right) \beta
$$

The parameter $k_{0}$ is introduced to allow for a zero point choice for the phase-step angle values. Without loss of generality, this zero point angle can be absorbed into the offset angle $\alpha$ and it is omitted in all the summations below.

In addition, for most, but not all, of the common phase step algorithms the coefficient angles $\delta_{k}$, Eq. (4), obey a linear relation similar to the phase step angles, Eq. (14), but with opposite sign (see the Appendix).

$$
\delta_{k}=\delta_{0}-k \delta .
$$

In this case, the constraints of Eq. (7) can be written in the form of polynomials ${ }^{10}$

$$
0=\sum_{k=0}^{N-1} c_{k} \exp \left(-i \beta_{k}\right)=\sum_{k=0}^{N-1}\left|c_{k}\right|[\exp (-i(\beta+\delta))]^{k}
$$

Similarly, if we consider the sequence of phase stepped signals as samples of a periodic signal $S(\tau)$ from which the phase should be retrieved, the discrete Fourier transform (DFT) of $S(\tau)$ is given by ${ }^{67}$

$$
\begin{aligned}
f(\nu)= & \frac{1}{N} \sum_{\tau=-N / 2}^{N / 2-1} S(\tau) e^{-i 2 \pi(\nu / N) \tau}=\frac{1}{N} \sum_{\tau=-N / 2}^{N / 2-1} S(\tau) \\
& \times \cos \left(2 \pi \frac{v}{N} \tau\right)+\frac{i}{N} \sum_{\tau=-N / 2}^{N / 2-1} S(\tau) \sin \left(-2 \pi \frac{v}{N} \tau\right) .
\end{aligned}
$$

Hence, for each Fourier component $f(v)$ the phase $\varphi(v)$ can be retrieved using the general Arctan algorithm, Eq. (2), with normalized coefficients $c_{k}=e^{-i 2 \pi(v / N) k}$ and equal phase step increments. Finally, when using a windowed Fourier transform approach, ${ }^{8,26,49}$ the coefficients $c_{k}$ are related to the sampling weights or windowing function $W_{k}$ by

$$
c_{k}=W_{k} e^{i k 2 \pi / N} .
$$

The assumptions used to arrive at the above results do not restrict the generality of the following analyses very much, as there are very few phase-reconstruction methods that do not adhere to them (see the Appendix).

\section{PHASE MEASUREMENT UNCERTAINTY FOR THE GENERAL ARCTAN ALGORITHM}

\section{A. Uncertainty analysis}

To perform the uncertainty analysis in accordance with the "Guide to the Expression of Uncertainty in Measurement" (GUM) ${ }^{58}$ we express the phase uncertainty in terms of individual signal uncertainties, $u\left(S_{k}\right)$, together with the correlations $u\left(S_{j}, S_{k}\right)$, because the measured signals $S_{k}$ are the experimental input quantities. The combined phase measurement uncertainty is calculated from Eq. (2) using error propagation $^{55}$ 


$$
\begin{aligned}
u^{2}\left(\varphi, S_{k}\right)= & \left(\frac{1}{1+\tan ^{2} \varphi}\right)^{2}\left\{\sum_{j=0}^{N-1}\left[\frac{\partial}{\partial S_{j}}\left(\frac{\mathbf{b} \cdot \mathbf{S}}{\mathbf{a} \cdot \mathbf{S}}\right)\right]^{2} u^{2}\left(S_{j}\right)\right. \\
& \left.+2 \sum_{j=0}^{N-1} \sum_{k>j}^{N-1}\left[\frac{\partial}{\partial S_{j}}\left(\frac{\mathbf{b} \cdot \mathbf{S}}{\mathbf{a} \cdot \mathbf{S}}\right)\right]\left[\frac{\partial}{\partial S_{k}}\left(\frac{\mathbf{b} \cdot \mathbf{S}}{\mathbf{a} \cdot \mathbf{S}}\right)\right] u\left(S_{j}, S_{k}\right)\right\}, \\
= & \frac{1}{G^{2}}\left\{\begin{array}{l}
\sum_{j=0}^{N-1}\left(b_{j} \cos \varphi-a_{j} \sin \varphi\right)^{2} u^{2}\left(S_{j}\right) \\
+2 \sum_{j=0}^{N-1} \sum_{k>j}^{N-1}\left(b_{j} \cos \varphi-a_{j} \sin \varphi\right)\left(b_{k} \cos \varphi-a_{k} \sin \varphi\right) u\left(S_{j}, S_{k}\right)
\end{array}\right\} .
\end{aligned}
$$

Introducing $G$ and angle $\delta_{j}$, Eqs. (4) and (9), the combined phase measurement uncertainty is simplified to

$$
u^{2}\left(\varphi, S_{k}\right)=\frac{4}{S_{\Gamma}^{2}\left|\mathbf{c} \cdot \mathbf{C}^{*}\right|^{2}}\left\{\begin{array}{l}
\sum_{j=0}^{N-1}\left|c_{j}\right|^{2} \sin ^{2}\left(\varphi-\delta_{j}\right) u^{2}\left(S_{j}\right) \\
+2 \sum_{j=0}^{N-1} \sum_{k>j}^{N-1}\left|c_{j}\right|\left|c_{k}\right| \sin \left(\varphi-\delta_{j}\right) \sin \left(\varphi-\delta_{k}\right) u\left(S_{j}, S_{k}\right)
\end{array}\right\} .
$$

This is the starting point for the error calculations that follow. Note that the first line in the curly brackets describes uncorrelated errors, while the second line describes correlations. The expression does not change when a rotated algorithm, Eq. (11), is used, except for the replacement of $\varphi$ by $\varphi+\gamma$.

The signal modulation $S_{\Gamma}$ in the denominator of Eq. (20) suggests that the phase measurement uncertainty can be expected to drop with increasing visibility, $v$, while the sine square expressions suggest that the combined phase measurement uncertainty is modulated at twice the angular frequency. Both statements, however, can become false if the uncertainties and correlations depend on $v$ or $\varphi$, respectively. Therefore we will group the influence factors according to their dependence on phase angle $\varphi$ and visibility $v$.

In order to calculate the covariance of the individual signals, $u\left(S_{j}, S_{k}\right)$, it is advantageous to explicitly introduce the parameters $p_{l}$ responsible for correlation, and their corresponding component of uncertainty ${ }^{58}$

$$
u\left(S_{j}, S_{k}\right)=\sum_{l}\left(\frac{\partial S_{j}}{\partial p_{l}}\right)\left(\frac{\partial S_{k}}{\partial p_{l}}\right) u^{2}\left(p_{l}\right) .
$$

\section{B. Uncertainty for the classical $\mathrm{N}$-bucket algorithm and Fourier method}

An important family of phase step algorithms is given by the classical $N$-bucket algorithms, ${ }^{10,47,63}$ also called synchronous detection algorithm, diagonal least-squares algorithm, ${ }^{26}$ or equal sampling algorithm, for which

$$
\beta_{k}=k \frac{2 \pi}{N}, \quad \mathbf{c}=\mathbf{C}^{*} .
$$

This same condition applies to Fourier transform methods as well with $N$ data points within one cycle. Equation (22) implies

$$
\begin{aligned}
\mathbf{a} \cdot \mathbf{a} & =\mathbf{b} \cdot \mathbf{b} \\
\mathbf{a} \cdot \mathbf{b} & =0
\end{aligned}, G=\frac{N}{2} S_{\Gamma}, \text { and } \quad \begin{aligned}
\delta_{k} & =-\beta_{k} \\
\left|c_{k}\right| & =1
\end{aligned} .
$$

Inserting Eqs. (22) and (23) into Eq. (20) yields the combined variance

$$
\begin{aligned}
u_{N}^{2}\left(\varphi, S_{k}\right)= & \frac{4}{N^{2} S_{\Gamma}^{2}}\left\{\sum_{k=0}^{N-1} \sin ^{2}\left(\varphi+\beta_{k}\right) u^{2}\left(S_{k}\right)\right. \\
& \left.+2 \sum_{j=0}^{N-1} \sum_{k>j}^{N-1} \sin \left(\varphi+\beta_{j}\right) \sin \left(\varphi+\beta_{k}\right) u\left(S_{j}, S_{k}\right)\right\} .
\end{aligned}
$$

The four-frame algorithm is the most widely used $\mathrm{N}$ bucket algorithm corresponding to $N=4$.

\section{CONTRIBUTIONS TO THE MEASUREMENT UNCERTAINTY}

Table I lists different uncertainty contributions to the phase measurement. In the following, their effect on the phase measurement uncertainty for a general Arctan algorithm, Eq. (20), for the $N$-bucket algorithm, Eq. (24), and the average contributions are given together with an upper bound. We thereby classify the contributions according to their correlation properties and phase angle dependence. 
TABLE I. Influence quantities and references to the respective formula for quantitative evaluation. Explicit expressions for algorithms with $\sin \left(\beta_{k}+\delta_{k}\right)=0 \forall k$ and for the $N$-bucket algorithms are included.

\begin{tabular}{|c|c|c|c|}
\hline Influence quantity to be estimated ${ }^{a}$ & General case & Phase angle average for cases with $\sin \left(\beta_{k}+\delta_{k}\right)=0 \forall k$ & $N$-bucket algorithms \\
\hline $\begin{array}{l}\text { Camera additive noise and quantization levels } \\
u(S)=S_{\text {camnoise }}(\mathrm{GL}) \\
u(S)=\frac{1}{\sqrt{12}} S_{\text {LSB }}(\mathrm{GL})\end{array}$ & Eq. (26) & $\begin{array}{c}\text { Eq. (27) } \\
\left\langle u^{2}\left(\varphi, S_{k}\right)\right\rangle_{\varphi}=2 \frac{u^{2}(S)}{S_{\Gamma}^{2}} F \\
F=\frac{|\mathbf{c}|^{2}}{\left|\mathbf{c} \cdot \mathbf{C}^{*}\right|^{2}}\end{array}$ & $\begin{array}{c}\text { Eq. (28) } \\
\begin{array}{c}u_{N}^{2}\left(\varphi, S_{k}\right)=\frac{u^{2}(S)}{S_{\Gamma}^{2}} \times \frac{2}{N} \\
F=\frac{1}{N}\end{array}\end{array}$ \\
\hline Illumination instability $r(I)(\%)$ & Eq. (31) & Eq. (32) & \\
\hline Camera gain fluctuations $r(\gamma)(\%)$ & & $\left\langle u^{2}(\varphi, \text { fluct })\right\rangle_{\varphi}=r^{2}().\left(\frac{2}{v^{2}}+\frac{1}{2}\right) F$ & $u_{N}^{2}(\varphi$, fluct $)=\frac{r^{2}(.)}{N}\left\{\frac{2}{v^{2}}+\frac{1}{2}-\left(\frac{2}{\nu} \delta_{N 3}+\frac{1}{2} \delta_{N 4}\right) \cos (N \varphi)\right\}$ \\
\hline Phase step jitter $u(\beta)(\mathrm{rad})$ & Eq. (34) & Eq. (35) $\left\langle u^{2}(\varphi, \beta)\right\rangle_{\varphi}=u^{2}(\beta) \frac{3}{2} F$ & $u_{N}^{2}(\varphi, \beta)=\frac{u^{2}(\beta)}{2 N}\left(3+\delta_{N 4} \cos (N \varphi)\right)$ \\
\hline $\begin{array}{l}\text { Poisson count statistics: number of photons } \\
S_{B}, S_{\Gamma} \text { (cnts) }\end{array}$ & Eq. (37) & $\begin{array}{c}\text { Eq. (38) } \\
\left\langle\left. u^{2}(\varphi, \text { Poisson })\right|_{\varphi}=\frac{2}{S_{B} v^{2}} F\right.\end{array}$ & $\begin{aligned} u_{N}^{2}(\varphi, \text { Poisson }) & =\frac{2}{S_{\Gamma}^{2} N}\left(S_{B}-\delta_{N 3} \frac{1}{2} S_{\Gamma} \cos (N \varphi)\right) \\
& =\frac{2}{S_{B} N v^{2}}\left(1-\delta_{N 3} \frac{1}{2} v \cos (N \varphi)\right)\end{aligned}$ \\
\hline $\begin{array}{l}\text { Linear phase step miscalibration } \beta(1+\varepsilon) \\
\varepsilon[\%]\end{array}$ & Eq. (41) & $\begin{array}{c}\text { Eq. (42) } \\
\left\langle u^{2}(\varphi, \varepsilon)\right\rangle_{\varphi} \leq \beta^{2} \varepsilon^{2} \frac{1}{4} N(N-1)(2 N-1) F\end{array}$ & $u_{N}(\varphi, \varepsilon)=\varepsilon \frac{\pi}{N}\left((N-1)-\frac{\sin \left(2 \varphi-\frac{2 \pi}{N}\right)}{\sin \left(\frac{2 \pi}{N}\right)}\right)$ \\
\hline $\begin{array}{l}\text { General phase step miscalibration } \\
\varepsilon_{k}=k \varepsilon \beta+k^{2} e_{2}+\cdots+k^{P} e_{P} e_{k}(\mathrm{rad})\end{array}$ & Eq. (44) & $\begin{array}{c}\text { Eq. (45) } \\
\left\langle u^{2}\left(\varphi, e_{p}\right)\right\rangle_{\varphi} \leq e_{p}^{2} \frac{3}{2} \sum_{k=0}^{N-1} k^{2 p} F\end{array}$ & $u_{N}\left(\varphi, e_{p}\right)=\frac{e_{p}}{N} \sum_{k=0}^{N-1} k^{p}\left(1-\cos \left(k \frac{4 \pi}{N}+2 \varphi\right)\right)$ \\
\hline $\begin{array}{l}\text { Detector nonlinearity } \tilde{S}_{k}=S_{k}\left(1+r_{2} S_{k}+\cdots+r_{J} S_{k}^{J-1}\right) \\
r_{n}\left(\mathrm{GL}^{1-\mathrm{n}}\right)\end{array}$ & Eq. (48) & & $\begin{array}{c}\text { lowest contributing order } N-1 \\
u_{N}^{2}\left(\varphi, r_{N-1}\right)=\left(r_{N-1} S_{B}^{N-2}\right)^{2}\left(\frac{v}{2}\right)^{2 N-4} \sin ^{2}(N \varphi)\end{array}$ \\
\hline Higher harmonics $\frac{S_{q}}{S_{\Gamma}}(\%)$ & Eq. (52) & Eq. (53) $\left\langle u^{2}\left(\varphi, S_{q}\right)\right\rangle_{\varphi} \leq \frac{S_{q}^{2}}{S_{\Gamma}^{2}} N F$ & $u_{N}^{2}\left(\varphi, S_{N-1}\right)=\frac{S_{N-1}^{2}}{S_{\Gamma}^{2}} \sin ^{2}(N \varphi)$ \\
\hline Background irradiance drift $\delta_{\text {drift }}(\%)$ & Eq. (56) & 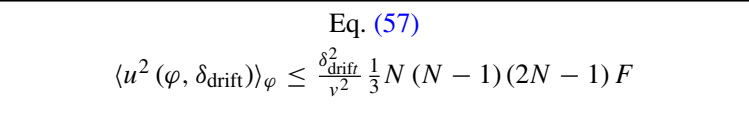 & $u_{N}^{2}\left(\varphi, \delta_{\text {drif } t}\right)=\frac{\delta_{\text {drift }}^{2}}{v^{2}}\left\{\frac{\cos ^{2}\left(\varphi-\frac{\pi}{N}\right)}{\sin ^{2}\left(\frac{\pi}{N}\right)}\right\}$ \\
\hline Source irradiance drift $\delta_{\text {illumdrift }}(\%)$ & Eq. (60) & $\begin{array}{c}\text { Eq. (61) } \\
\left\langle u^{2}\left(\varphi, \delta_{\text {illum.drift } t)}\right) \leq \delta_{\text {illum.drift }}^{2}\left(\frac{1}{v^{2}}+\frac{1}{4}\right) \frac{1}{3} N(N-1)(2 N-1) F\right.\end{array}$ & $\begin{array}{c}u_{N}^{2}\left(\varphi, \delta_{\text {illum.drift }}\right) \\
=\delta_{\text {illum.drift } t}^{2}\left\{\frac{1}{v} \frac{\cos \left(\varphi-\frac{\pi}{N}\right)}{\sin \left(\frac{\pi}{N}\right)}+\frac{\cos \left(2 \varphi-\frac{2 \pi}{N}\right)}{2 \sin \left(\frac{2 \pi}{N}\right)}\right\}^{2}\end{array}$ \\
\hline
\end{tabular}

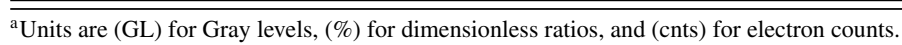




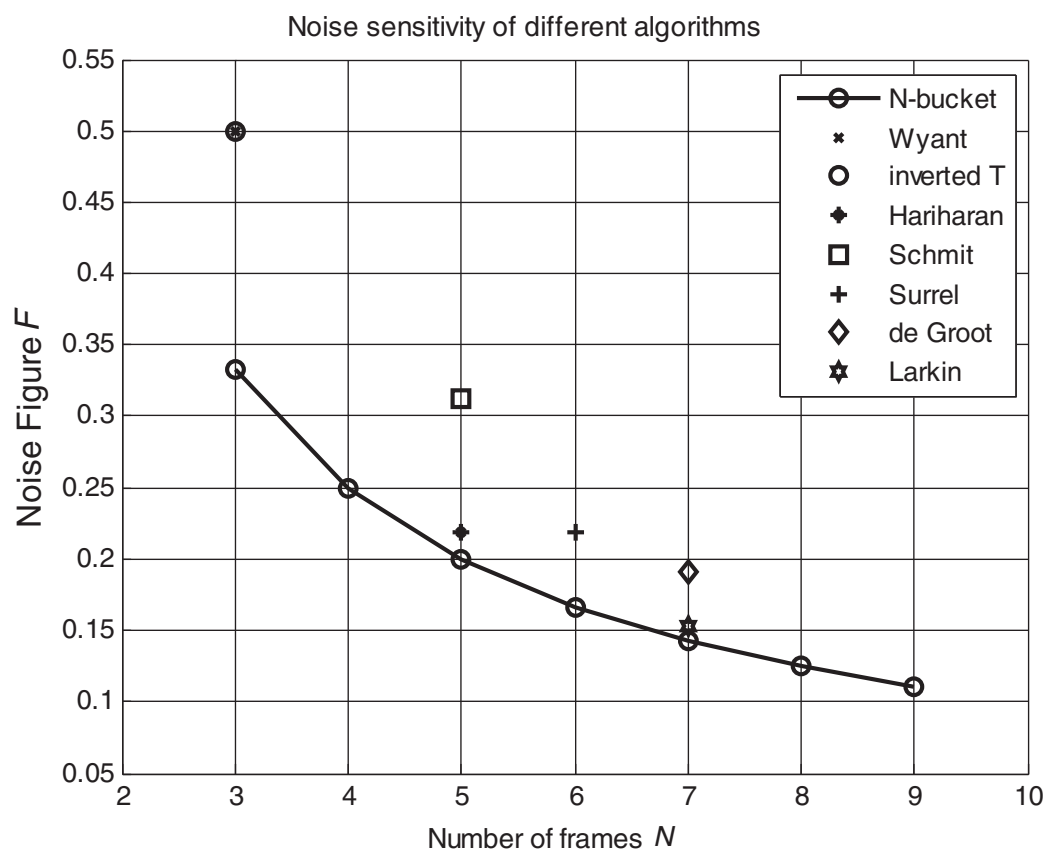

FIG. 4. Sensitivity of different phase stepping algorithms as expressed with the noise figure F. For definitions of the names used, see the Appendix.

\section{A. Uncorrelated, phase-independent contributions}

Additive camera noise, incoherent background illumination noise, and digitization jitter are contributions that are uncorrelated, phase-independent, and equal for all signals $S_{k}$, i.e., $u^{2}\left(S_{k}\right)=u^{2}(S)$ and $u\left(S_{j}, S_{k}\right)=0$ for $j \neq k$. In a CCD or CMOS sensor, additive signal noise $\mathrm{C}^{1,11,56,68-71}$ is caused by the reset noise, the electronic thermal noise of the readout circuit, and the shot noise of the dark current. Let $S_{\text {cam.noise }}^{2}$ be the variance describing the camera noise, $S_{\text {illum.noise }}^{2}$ the variance of the background illumination, and $S_{\mathrm{LSB}}$ the width of the least significant bit (LSB). Because the signal uncertainty due to the LSB is distributed according to a rectangular probability density function, the variance is obtained by multipling one twelfth to the square of the width. The variances are added up to yield

$$
u^{2}(S)=S_{\text {cam.noise }}^{2}+S_{\text {illum.noise }}^{2}+\frac{1}{12} S_{\mathrm{LSB}}^{2} .
$$

The digitization jitter or quantization noise, $S_{\mathrm{LSB}}{ }^{47,55,56,72-74}$ can normally be neglected against the electronic additive noise, if the digitization depth is 8 bit or more. ${ }^{71}$ One may take into account correlations between the quantization errors, ${ }^{55}$ if more than one cycle is sampled during the phase stepping procedure. However, the effect normally is negligible.

We have from Eq. (20), 1,20,26

$$
\begin{aligned}
u^{2}\left(\varphi, S_{k}\right)= & \frac{4 u^{2}(S)}{S_{\Gamma}^{2}\left|\mathbf{c} \cdot \mathbf{C}^{*}\right|^{2}} \sum_{j=0}^{N-1}\left|c_{j}\right|^{2} \sin ^{2}\left(\varphi-\delta_{j}\right) \\
= & \frac{2 u^{2}(S)}{S_{\Gamma}^{2}\left|\mathbf{c} \cdot \mathbf{C}^{*}\right|^{2}}\left\{|\mathbf{c}|^{2}-(\mathbf{a} \cdot \mathbf{a}-\mathbf{b} \cdot \mathbf{b})\right. \\
& \times \cos (2 \varphi)-2 \mathbf{a} \cdot \mathbf{b} \sin (2 \varphi)\}
\end{aligned}
$$

The uncertainty is modulated with twice the phase angle, except for algorithms for which both $\mathbf{a} \cdot \mathbf{b}=0$ and $\mathbf{a} \cdot \mathbf{a}$ = b $\cdot \mathbf{b}$, such as the classical $N$-bucket algorithm, Eq. (23). Their phase measurement uncertainty coincides with the uncertainty of a general Arctan algorithm averaged over all phase angles $26,55,56,63,70$

$$
\begin{gathered}
\left\langle u^{2}\left(\varphi, S_{k}\right)\right\rangle_{\varphi}=2 \frac{u^{2}(S)}{S_{\Gamma}^{2}} F, \\
F=\frac{|\mathbf{c}|^{2}}{\left|\mathbf{c} \cdot \mathbf{C}^{*}\right|^{2}} .
\end{gathered}
$$

In this case, the factor $F \geq 1 / N$ qualifies as a noise figure. ${ }^{11}$ Note also that it is related to the loss factor $\eta$ described in Ref. 68 via $F=1 / N \eta^{2}$, so that $F$ is an absolute figure of merit and $\eta$ is a relative one. The lower limit derives from Eq. (10) and is reached by the $N$-bucket algorithms ${ }^{20,47,65}$ for which

$$
u_{N}^{2}\left(\varphi, S_{k}\right)=\frac{u^{2}(S)}{S_{\Gamma}^{2}} \frac{2}{N},
$$

so that $u_{N}$ drops with $\sqrt{N}$ and with increasing signal modulation $S_{\Gamma}$. Figure 4 shows the sensitivity of several phase stepping algorithms, expressed through the noise figure $F$, Eq. (27).

\section{B. Uncorrelated contributions involving the phase angle}

\section{Illumination instability and camera gain fluctuations}

If the illuminating light source is not stable during the phase stepping procedure, it induces an irradiance fluctuation proportional to the signal itself. ${ }^{1,47}$ It has the same effect as 


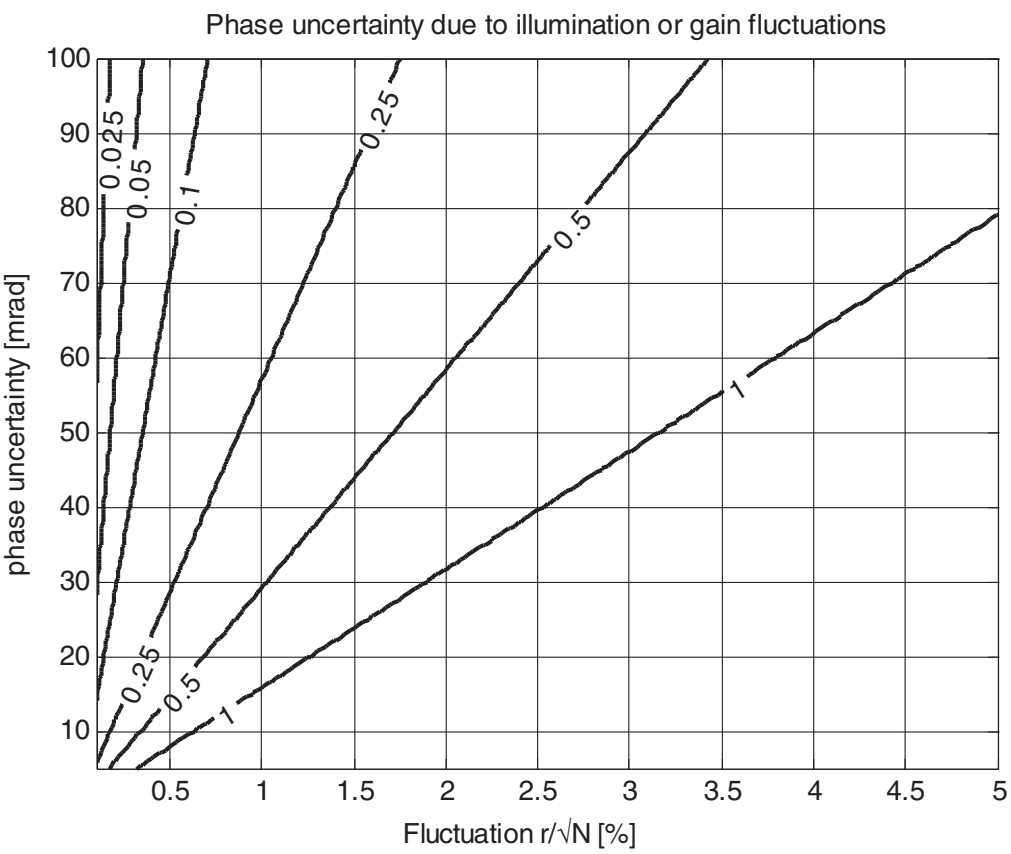

FIG. 5. Average phase uncertainty [mrad] as a function of fluctuation $r$ in illumination intensity or camera gain for $N$-bucket algorithms. Lines are plotted for different values of the visibility.

a gain variation from frame to frame, i.e., multiplicative camera noise. Correlation between the frames can be neglected if the fluctuations are faster than the frame acquisition rate. Otherwise, the intensity is regarded as drifting (see below).

According to Eq. (1) both contributions have the same effect. Introducing $r($.$) as the relative uncertainty of the re-$ spective input parameter, we can write

$$
\begin{aligned}
& u^{2}\left(S_{k}, \gamma\right)=I_{k}^{2} u^{2}(\gamma)=S_{k}^{2} r^{2}(\gamma), \\
& u^{2}\left(S_{k}, I_{\text {fluct }}\right)=\gamma^{2} u^{2}\left(I_{k}\right)=S_{k}^{2} r^{2}(I) .
\end{aligned}
$$

From Eq. (20) this leads to

$$
u^{2}(\varphi, \text { fluct })=4 \frac{r^{2}(.)}{S_{\Gamma}^{2}\left|\mathbf{c} \cdot \mathbf{C}^{*}\right|^{2}} \sum_{k=0}^{N-1}\left|c_{k}\right|^{2} \sin ^{2}\left(\varphi-\delta_{k}\right) S_{k}^{2} .
$$

Inserting the signal decomposition into Eq. (30) yields

$$
\begin{aligned}
u^{2}(\varphi, \text { fluct })= & \frac{r^{2}(.)}{\left|\mathbf{c} \cdot \mathbf{C}^{*}\right|^{2}} 4 \sum_{k=0}^{N-1}\left|c_{k}\right|^{2} \sin ^{2}\left(\varphi-\delta_{k}\right) \\
& \times\left\{\frac{S_{B}^{2}}{S_{\Gamma}^{2}}+2 \frac{S_{B}}{S_{\Gamma}} \cos \left(\varphi+\beta_{k}\right)+\cos ^{2}\left(\varphi+\beta_{k}\right)\right\} .
\end{aligned}
$$

These fluctuations are a severe source of phase uncertainty in cases where the modulation signal is small compared to the background signal, i.e., the fringe visibility is poor. The average phase angle error is found from Eq. (31) after isolating the phase angle dependent terms,

$$
\left\langle u^{2}(\varphi, \text { fluct })\right\rangle_{\varphi}=r^{2}(.)\left\{2 \frac{S_{B}^{2}}{S_{\Gamma}^{2}}+\frac{1}{2}\right\} \frac{|\mathbf{c}|^{2}}{\left|\mathbf{c} \cdot \mathbf{C}^{*}\right|^{2}}+\frac{r^{2}(.)}{\left|\mathbf{c} \cdot \mathbf{C}^{*}\right|^{2}}
$$

$$
\begin{aligned}
& \times \sum_{k=0}^{N-1}\left|c_{k}\right|^{2} \sin ^{2}\left(\delta_{k}+\beta_{k}\right), \\
\leq & r^{2}(.)\left\{\frac{2}{v^{2}}+\frac{3}{2}\right\} F,
\end{aligned}
$$

where again the noise figure $F$, Eq. (27), appears. For the $N$ bucket algorithm ${ }^{47,56}$ the sums in Eq. (31) can be analytically evaluated, and the result (see Table I) is illustrated in Fig. 5. We further note that for most algorithms $\sin \left(\delta_{k}+\beta_{k}\right)=0$ (the Appendix) for which the average uncertainty is given in Table I.

\section{Phase step jitter}

If the phase step angle $\beta_{k}$ is randomly jittering around its expectation value with a standard deviation of $u(\beta)$, this entails a phase measurement uncertainty. The same is true for random object vibrations that induce a jitter in the phase. From Eq. (1) the signal uncertainty due to phase step (or object phase) jitter is obtained according to

$$
u^{2}\left(S_{k}, \beta_{k}\right)=S_{\Gamma}^{2} \sin ^{2}\left(\varphi+\beta_{k}\right) u^{2}\left(\beta_{k}\right)
$$

Note that the uncertainty is proportional to the signal modulation $S_{\Gamma}$ and depends on the phase angle $\varphi$, even if we assume that the phase step jitter itself is independent of $k$ and uncorrelated, $u^{2}\left(\beta_{k}\right)=u^{2}(\beta)$. Introducing this expression into the general expression, Eq. (20), we have

$$
u^{2}(\varphi, \beta)=\frac{u^{2}(\beta)}{\left|\mathbf{c} \cdot \mathbf{C}^{*}\right|^{2}} 4 \sum_{k=0}^{N-1}\left|c_{k}\right|^{2} \sin ^{2}\left(\varphi-\delta_{k}\right) \sin ^{2}\left(\varphi+\beta_{k}\right) \text {. }
$$




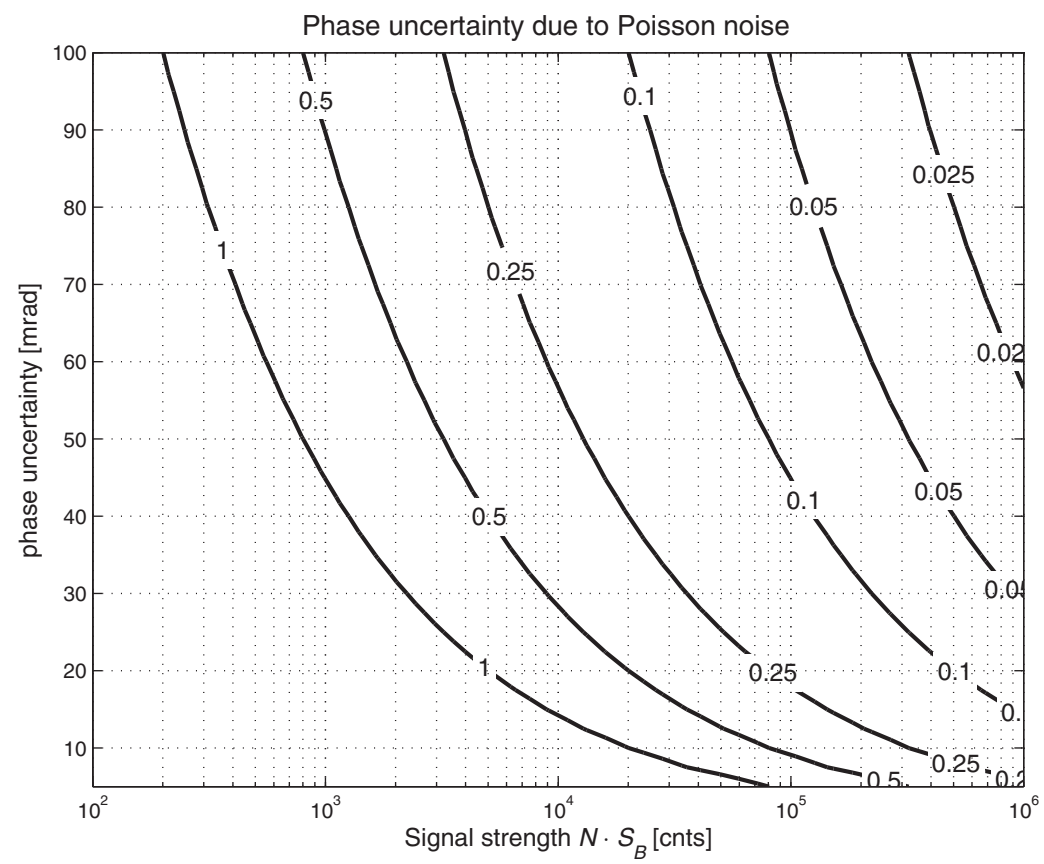

FIG. 6. Phase uncertainty in [mrad] due to shot noise for $N$-bucket algorithms, as a function of the number of counts. Lines are plotted for different values of the visibility.

Phase step jitter is a critical source of measurement uncertainty, as it cannot be reduced by increasing the signal modulation. After averaging over the phase angles, Eq. (34) reduces to ${ }^{20}$

$$
\begin{aligned}
\left\langle u^{2}(\varphi, \beta)\right\rangle_{\varphi}= & \frac{u^{2}(\beta)}{\left|\mathbf{c} \cdot \mathbf{C}^{*}\right|^{2}} \sum_{k=0}^{N-1}\left|c_{k}\right|^{2} \frac{1}{2}\left(1+2 \cos ^{2}\left(\delta_{k}+\beta_{k}\right)\right) \\
& \leq u^{2}(\beta) \frac{3}{2} F .
\end{aligned}
$$

The upper limit is reached for most of the techniques in the Appendix, due to the fact that $\cos \left(\delta_{k}+\beta_{k}\right)=1$. For the $N$-bucket algorithm, Eq. (34) can be analytically evaluated (see Table I). Only the case $N=4$ has been reported before. ${ }^{56}$

\section{Poisson count statistics}

In a photon detector (CCD and CMOS cameras, photon counter) the number of counts per exposure varies according to Poisson statistics (shot noise). The maximum number of electrons a pixel can hold is given by the full well depth. Typical values are less than $10^{5}$ electrons per pixel, corresponding to a shot noise level of at least $0.3 \%$. The variance of the signal is proportional to the signal itself ${ }^{1,71,75,76}$ when signals are measured in counts (cnts)

$$
u^{2}\left(S_{k}\right)=S_{B}+S_{\Gamma} \cos \left(\varphi+\beta_{k}\right) .
$$

Introducing this Poisson noise into Eq. (20), we have

$$
u^{2}(\varphi, \text { Poisson })=\frac{4}{S_{\Gamma}^{2}\left|\mathbf{c} \cdot \mathbf{C}^{*}\right|^{2}}
$$

$$
\times\left(\begin{array}{l}
S_{B} \sum_{k=0}^{N-1}\left|c_{k}\right|^{2} \sin ^{2}\left(\varphi-\delta_{k}\right) \\
+S_{\Gamma} \sum_{k=0}^{N-1}\left|c_{k}\right|^{2} \sin ^{2}\left(\varphi-\delta_{k}\right) \cos \left(\varphi+\beta_{k}\right)
\end{array}\right) .
$$

The uncertainty averaged over the phase angle is ${ }^{11}$

$$
\left\langle u^{2}(\varphi, \text { Poisson })\right\rangle_{\varphi}=2 \frac{S_{B}}{S_{\Gamma}^{2}} F=\frac{2}{S_{B} v^{2}} F,
$$

again proportional to the noise figure $F$, Eq. (27), but inversely proportional to the square of the visibility. For the $\mathrm{N}$ bucket algorithm, Eq. (37) can be analytically evaluated (see Table I), and the results are depicted in Fig. 6.

\section{Correlated contributions}

\section{Linear phase step miscalibration}

If the phase step angle $\beta$ does not exactly match the design phase step, there is a systematic phase angle deviation, sometimes also called detuning or slope error. ${ }^{3,6,7,14,18,19,28,30,31,47,51,56,66,77,78}$ For the case of a linear phase step miscalibration, we have from Eq. (1)

$$
\begin{aligned}
\tilde{S}_{k} & =S_{B}+S_{\Gamma} \cos (\varphi+k \beta(1+\varepsilon)), \\
\left.\frac{\partial \tilde{S}_{k}}{\partial \varepsilon}\right|_{\varepsilon=0} & =-S_{\Gamma} \sin (\varphi+k \beta) k \beta,
\end{aligned}
$$

where $\varepsilon$ is the relative step angle error. The deviation increases linearly with $k$ and is again proportional to the modulation signal. As a consequence, the phase measurement uncertainty due to a linear phase step miscalibration cannot be reduced by increasing the signal modulation $S_{\Gamma}$. The miscalibration coefficient $\varepsilon$ may vary across an image. It introduces a systematic phase variation which is not known 
a priori (otherwise one could correct for it), and therefore it is treated as a measurement uncertainty like a random uncertainty contribution. ${ }^{58}$ The correlation of the signals must be taken into account according to Eq. (21)

$$
\begin{aligned}
u^{2}\left(S_{k}, \varepsilon\right) & =S_{\Gamma}^{2} \sin ^{2}(\varphi+k \beta) k^{2} \beta^{2} \varepsilon^{2}, \\
u_{\varepsilon}\left(S_{j}, S_{k}\right) & =S_{\Gamma}^{2} \sin (\varphi+j \beta) \sin (\varphi+k \beta) j k \beta^{2} \varepsilon^{2},
\end{aligned}
$$

Insertion into the general expression Eq. (20) leaves us with

$$
u^{2}(\varphi, \varepsilon)=\frac{\beta^{2} \varepsilon^{2}}{\left|\mathbf{c} \cdot \mathbf{C}^{*}\right|^{2}} 4\left\{\sum_{k=0}^{N-1}\left|c_{k}\right| k \times \sin \left(\varphi-\delta_{k}\right) \sin \left(\varphi+\beta_{k}\right)\right\}^{2} .
$$

The phase measurement uncertainty due to a linear phase step miscalibration is therefore independent of the signal level and the signal modulation. The average phase measurement uncertainty and its upper bound are

$$
\begin{aligned}
\left\langle u^{2}(\varphi, \varepsilon)\right\rangle_{\varphi}= & \frac{\beta^{2} \varepsilon^{2}}{\left|\mathbf{c} \cdot \mathbf{C}^{*}\right|^{2}}\left\langle\left\{\sum_{k=0}^{N-1}\left|c_{k}\right| k \cos \left(\delta_{k}+\beta_{k}\right)\right\}^{2}\right. \\
& +\left\{\sum_{k=0}^{N-1}\left|c_{k}\right| k \cos \left(2 \varphi+\beta_{k}-\delta_{k}\right)\right\}^{2}, \\
& \leq \beta^{2} \varepsilon^{2} F\left\{\sum_{k=0}^{N-1} k^{2} \cos ^{2}\left(\delta_{k}+\beta_{k}\right)\right. \\
& \left.+\left\langle\sum_{k=0}^{N-1} k^{2} \cos ^{2}\left(2 \varphi+\beta_{k}-\delta_{k}\right)\right\rangle\right\}, \\
& \leq \beta^{2} \varepsilon^{2} F \frac{3}{2} \sum_{k=0}^{N-1} k^{2}=\beta^{2} \varepsilon^{2} \frac{1}{4} N(N-1)(2 N-1) F .
\end{aligned}
$$

The uncertainty for the $N$-bucket algorithm is calculated from inserting Eq. (22) into Eq. (41) and is given in Table I. ${ }^{10,19,49,53,56}$ For $N=4$ we find $u_{N}(\varphi, \varepsilon)$ $=\varepsilon^{\pi} / 4(3-\cos (2 \varphi))$.

\section{General phase step miscalibration}

The general phase step uncertainty will include not only linear but also higher order terms. For a general phase step miscalibration, a power series in $k$ up to the order $P$ may be assumed

$$
\begin{aligned}
\beta_{k}^{e f f} & =k \beta(1+\varepsilon)+p_{2}(k \beta)^{2}+\ldots+p_{P}(k \beta)^{P}, \\
\varepsilon_{k} & =k \varepsilon \beta+k^{2} e_{2}+\ldots+k^{P} e_{P} .
\end{aligned}
$$

Hence, in analogy to Eq. (40) when replacing the linear miscalibration effect $k \varepsilon \beta$ by the full $\varepsilon_{k}$ the deviation is again proportional to the signal modulation when the effect is treated in linear approximation. ${ }^{20,22,29,31,56,70,79}$ Since we do not know the actual values of the polynomial coefficients, $e_{p}$, in a statistical sense all the cross-terms involving $e_{p} e_{q}$ with $p \neq q$ vanish on average, and we are left with only quadratic terms in $e_{p}$ both for the uncertainty terms $\varepsilon_{k}^{2}$ and $\varepsilon_{j} \varepsilon_{k}$. This allows treating each order independently, and we have from Eq. (20)

$$
\begin{aligned}
u^{2}\left(\varphi, e_{p}\right)= & \frac{e_{p}^{2}}{\left|\mathbf{c} \cdot \mathbf{C}^{*}\right|^{2}} 4 \\
& \times\left\{\sum_{k=0}^{N-1}\left|c_{k}\right| k^{p} \sin \left(\delta_{k}-\varphi\right) \sin \left(\varphi+\beta_{k}\right)\right\}^{2} .
\end{aligned}
$$

The average phase measurement uncertainty is bounded from above by

$$
\left\langle u^{2}\left(\varphi, e_{p}\right)\right\rangle_{\varphi} \leq e_{p}^{2} \frac{3}{2} \sum_{k=0}^{N-1} k^{2 p} F .
$$

The uncertainty for the $N$-bucket algorithm is analytically obtained up to a sum containing $k^{p}$ and a cosine of $2 \varphi$ (see Table I). A closed form solution is cumbersome, but the average uncertainty scales with $N^{p-1}$ showing that uncertainty caused by higher order nonlinearities increases steeply with the number of frames.

\section{Detector nonlinearity}

Detector nonlinearity is a systematic error that causes higher harmonics and correlates the individual signals. ${ }^{29,42,47,51,56,80-82}$ Due to the ubiquitous use of CMOS sensors (which are slightly nonlinear, and inferior to CCDs in this regard), this effect has regained attention today. Let the ideal signal, Eq. (1), be replaced by

$$
\begin{aligned}
\tilde{S}_{k} & =\gamma I_{k}+\gamma_{2} I_{k}^{2}+\cdots+\gamma_{J} I_{k}^{J} \\
& =S_{k}\left(1+r_{2} S_{k}+\cdots+r_{J} S_{k}^{J-1}\right), \\
r_{n} & =\frac{\gamma_{n}}{\gamma^{n}}, \\
\frac{\partial \tilde{S}_{k}}{\partial r_{n}} & =S_{k}^{n},
\end{aligned}
$$

where $J$ is the highest order of detector nonlinearity and $r_{n}$ is the relative nonlinearity parameter for order $n$. Since we do not know the actual values of the polynomial coefficients, $r_{n}$, in a statistical sense all the cross-terms $r_{n} r_{m}$ involving $n \neq m$ vanish on average. As explained above, this then allows discussing the nonlinear contributions for each order separately. The uncertainty components are thus

$$
u^{2}\left(S_{k}, r_{n}\right)=S_{k}^{2 n} r_{n}^{2} \quad u\left(S_{j}, S_{k}, r_{n}\right)=S_{j}^{n} S_{k}^{n} r_{n}^{2} .
$$

The signal errors are fully correlated because for each $n$, the detector nonlinearity affects all individual signals likewise. From Eq. (20) we have

$u^{2}\left(\varphi, r_{n}\right)=\frac{r_{n}^{2}}{S_{\Gamma}^{2}\left|\mathbf{c} \cdot \mathbf{C}^{*}\right|^{2}} 4\left\{\sum_{k=0}^{N-1}\left|c_{k}\right| \sin \left(\varphi-\delta_{k}\right) S_{k}^{n}\right\}^{2}$. 


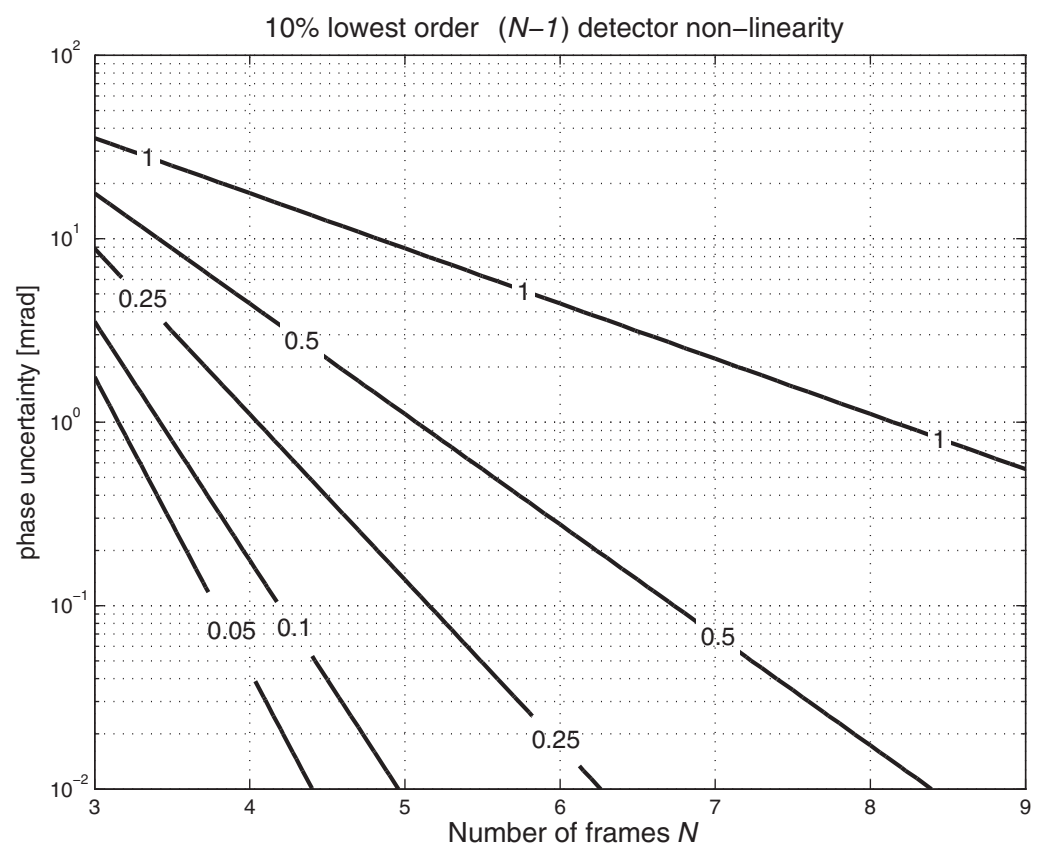

FIG. 7. Average phase uncertainty due to $10 \%$ lowest contributing order $(N-1)$ detector nonlinearity, i.e., $\left(r_{N-1} S_{B}^{N-2}\right)=0.1$ as a function of the number of frames of the $N$-bucket algorithm, Table I. Lines are plotted for different values of the visibility.

Decomposing the signal into its background and modulation, we obtain

$$
\begin{aligned}
& \sum_{k=0}^{N-1}\left|c_{k}\right| \sin \left(\varphi-\delta_{k}\right) S_{k}^{n}=S_{B}^{n} \sum_{m=0}^{n}\left(\begin{array}{c}
n \\
m
\end{array}\right) v^{m} \\
& \quad \times \sum_{k=0}^{N-1}\left|c_{k}\right| \sin \left(\varphi-\delta_{k}\right) \cos ^{m}\left(\varphi+\beta_{k}\right) .
\end{aligned}
$$

The general sum involving the power $m$ of the cosine cannot be simplified in general, while the respective expression for the $\mathrm{N}$-bucket algorithm can be evaluated analytically. For the latter we find that the sum over the powers of the cosine vanishes, except for orders $n=m=N-1$ and above. The resulting uncertainty is given in Table I for the lowest contributing order (Fig. 7). This result generalizes the result of van Wingerden et al. ${ }^{56}$ who - in case of the 4-frame algorithm - finds a proportionality to $\sin (4 \varphi)$, and a vanishing contribution for quadratic detector nonlinearity. Note that the uncertainty increases with increase in the fringe contrast.

\section{Higher harmonics}

Higher harmonics in the signal can be caused, e.g., by the use of Ronchi rulings or uncalibrated projectors in fringe projection, by Talbot projection in shadow moiré, by interference of multiple laser beams, or by signal clipping due to camera saturation. They lead to additional terms in the measured signal

$$
\tilde{S}_{k}=S_{k}+\sum_{q=2}^{Q} S_{q} \cos \left(q\left(\varphi+\beta_{k}\right)\right)
$$

If nothing is known about the higher harmonics present in the signal, i.e., no Fourier decomposition is known, then these orders are taken to be uncorrelated and are treated individually. The sensitivity to order $q$ is given by

$$
\frac{\partial \tilde{S}_{k}}{\partial S_{q}}=\cos \left(q\left(\varphi+\beta_{k}\right)\right)
$$

which leads again to a full correlation of all signals within a given harmonic order $q$. Hence, in analogy to Eq. (48), the uncertainty is given by

$$
\begin{aligned}
u^{2}\left(\varphi, S_{q}\right)= & \frac{S_{q}^{2}}{S_{\Gamma}^{2}\left|\mathbf{c} \cdot \mathbf{C}^{*}\right|^{2}} 4 \\
& \times\left\{\sum_{k=0}^{N-1}\left|c_{k}\right| \sin \left(\varphi-\delta_{k}\right) \cos \left(q\left(\varphi+\beta_{k}\right)\right)\right\}^{2} .
\end{aligned}
$$

The uncertainty is directly proportional to the ratio of the higher-order signal content $S_{q}$ to the fundamental-frequency content of the signal $S_{\Gamma}$. The general sum involving the cosine of the $q$ th harmonic cannot be simplified further. Because $\cos (q \varphi)$ can be decomposed into powers of $\cos (\varphi)$ up to the order $q$, we have a similar situation as with the detector nonlinearity above. The sum for the $N$-bucket algorithm vanishes again, except for the cases when $q \pm 1=Z N$, where $Z$ is an integer. The lowest contributing order for a given $N$ is $q=N-1$ and the next higher order is $q=N+1$. Their contribution is given by the identical expression (see Table I). Again, the case of the 4-frame algorithm with a proportionality to $\sin (4 \varphi)$ was reported before (e.g., Ref. 83 ). Hence, if 
insensitivity to the harmonics up to the $q$ th order that are present in the signal is required, the minimal number of signal values required is $N=q+2 \cdot{ }^{10,49,52}$

For the general case, the averaged variance can be estimated by

$$
\begin{aligned}
\left\langle u^{2}\left(\varphi, S_{q}\right)\right\rangle_{\varphi} & \leq \frac{S_{q}^{2}}{S_{\Gamma}^{2}} 4 F\left\{\sum_{k=0}^{N-1}\left\langle\sin ^{2}\left(\varphi-\delta_{k}\right) \cos ^{2}\left(q\left(\varphi+\beta_{k}\right)\right)\right\rangle_{\varphi}\right\} \\
& =\frac{S_{q}^{2}}{S_{\Gamma}^{2}} N F
\end{aligned}
$$

\section{Drift of background irradiance}

If the background irradiance is linearly drifting, also called bias modulation, during phase stepping, ${ }^{68,84,85}$ we have from Eq. (1)

$$
\begin{aligned}
\tilde{S}_{k} & =S_{B}\left(1+k \delta_{d r i f t}\right)+S_{\Gamma} \cos (\varphi+k \beta), \\
\left.\frac{\partial \tilde{S}_{k}}{\partial \delta_{\text {drift }}}\right|_{\varepsilon=0} & =k S_{B} .
\end{aligned}
$$

The deviation increases linearly with $k$, but it is not modulated. Since $\delta_{\text {drift }}$ is unknown it must be treated as an uncertainty.

$$
\begin{aligned}
& u^{2}\left(S_{k}, \delta_{\text {drift }}\right)=k^{2} S_{B}^{2} \delta_{\text {drift }}^{2}, \\
& u_{\text {drift }}\left(S_{j}, S_{k}\right)=S_{B}^{2} j k \delta_{\text {drift }}^{2} .
\end{aligned}
$$

Inserting into the general expression, Eq. (20), we get, observing the full correlation

$$
u^{2}\left(\varphi, \delta_{\mathrm{drif} t}\right)=\frac{S_{B}^{2} \delta_{\mathrm{drif} t}^{2}}{S_{\Gamma}^{2}\left|\mathbf{c} \cdot \mathbf{C}^{*}\right|^{2}} 4\left\{\sum_{k=0}^{N-1}\left|c_{k}\right| \cdot k \cdot \sin \left(\varphi-\delta_{k}\right)\right\}^{2} .
$$

Drift in the background irradiance is therefore a major source of uncertainty, because it is inversely proportional to the fringe contrast. The upper bound for the average measurement uncertainty is from Eq. (56),

$$
\begin{aligned}
\left\langle u^{2}\left(\varphi, \delta_{\text {drift }}\right)\right\rangle_{\varphi}= & \frac{S_{B}^{2} \delta_{\text {drift }}^{2}}{S_{\Gamma}^{2}\left|\mathbf{c} \cdot \mathbf{C}^{*}\right|^{2}} 2\left[\left\{\sum_{k=0}^{N-1}\left|c_{k}\right| \cdot k \cdot \sin \left(\delta_{k}\right)\right\}^{2}\right. \\
& \left.+\left\{\sum_{k=0}^{N-1}\left|c_{k}\right| \cdot k \cdot \cos \left(\delta_{k}\right)\right\}^{2}\right] \\
\left\langle u^{2}\left(\varphi, \delta_{\text {drift } t}\right)\right\rangle_{\varphi} \leq & \frac{S_{B}^{2} \delta_{\text {drift }}^{2} 2 F \sum_{k=0}^{N-1} k^{2}}{S_{\Gamma}^{2}} \\
= & \frac{\delta_{\text {drift }}^{2}}{v^{2}} \frac{1}{3} N(N-1)(2 N-1) F
\end{aligned}
$$

For the $N$-bucket algorithm the evaluated sum from Eq. (56) is given in Table I.

\section{Drift of source irradiance}

In case of a linear drift in the illumination source irradiance, the entire signal (background and modulation) is affected. This can happen, e.g., during wavelength-shifting when the source power cannot be kept constant. ${ }^{84,85}$ The induced uncertainty component is

$$
\begin{aligned}
& u^{2}\left(S_{k}, I_{\text {drif } t}\right)=k^{2} S_{k}^{2} \delta_{\text {illum.drift }}^{2}, \\
& u_{\text {drift } t}\left(S_{j}, S_{k}\right)=S_{j} S_{k} j k \delta_{\text {illum.drift }}^{2} .
\end{aligned}
$$

The contribution to the phase measurement uncertainty is

$$
u^{2}\left(\varphi, I_{\text {drif } t}\right)=\frac{\delta_{\text {illum.drift }}^{2}}{S_{\Gamma}^{2}\left|\mathbf{c} \cdot \mathbf{C}^{*}\right|^{2}} 4\left\{\sum_{k=0}^{N-1}\left|c_{k}\right| \sin \left(\varphi-\delta_{k}\right) k S_{k}\right\}^{2}
$$

Inserting the signal decomposition, Eq. (1), we obtain

$$
\begin{aligned}
u^{2}\left(\varphi, \delta_{\text {illum.drift }}\right)= & \frac{\delta_{\text {illum.drift }}^{2}}{\left|\mathbf{c} \cdot \mathbf{C}^{*}\right|^{2}}\left\{\frac{S_{B}}{S_{\Gamma}} \sum_{k=0}^{N-1}\left|c_{k}\right| \sin \left(\varphi-\delta_{k}\right) k\right. \\
& \left.+\sum_{k=0}^{N-1}\left|c_{k}\right| \sin \left(\varphi-\delta_{k}\right) k \cos \left(\varphi+\beta_{k}\right)\right\}^{2},
\end{aligned}
$$

and the upper bound for the uncertainty averaged over the phase angle

$$
\begin{aligned}
& \left\langle u^{2}\left(\varphi, \delta_{\text {illum.drift }}\right)\right\rangle_{\varphi} \leq \delta_{\text {illum.drift }}^{2} 2 F\left\{\left(\frac{S_{B}^{2}}{S_{\Gamma}^{2}}+\frac{1}{4}\right)\left(\sum_{k=0}^{N-1} k^{2}\right)\right. \\
& \left.\quad+\frac{1}{2}\left(\sum_{k=0}^{N-1} \sin ^{2}\left(\delta_{k}+\beta_{k}\right) k^{2}\right)\right\}, \\
& \leq \delta_{\text {illum.drift }}^{2}\left(\frac{1}{v^{2}}+\frac{3}{4}\right) \frac{1}{3} N(N-1)(2 N-1) F .
\end{aligned}
$$

The analytic expression for the $N$-bucket algorithm is given in Table I.

\section{COMBINED PHASE MEASUREMENT UNCERTAINTY}

All uncertainty contributions must be added in order to obtain the combined measurement uncertainty of the phase measurement algorithm. From the discussion in the previous chapter, it is best to sort the contributions by their dependence on the number of frames $N$ and the fringe contrast $v$. For lack of space, we only give the combined measurement uncertainty for the $N$-bucket algorithms averaged over the phase angles 
$1 \%$ equivalent uncertainty contributions, $v=1$

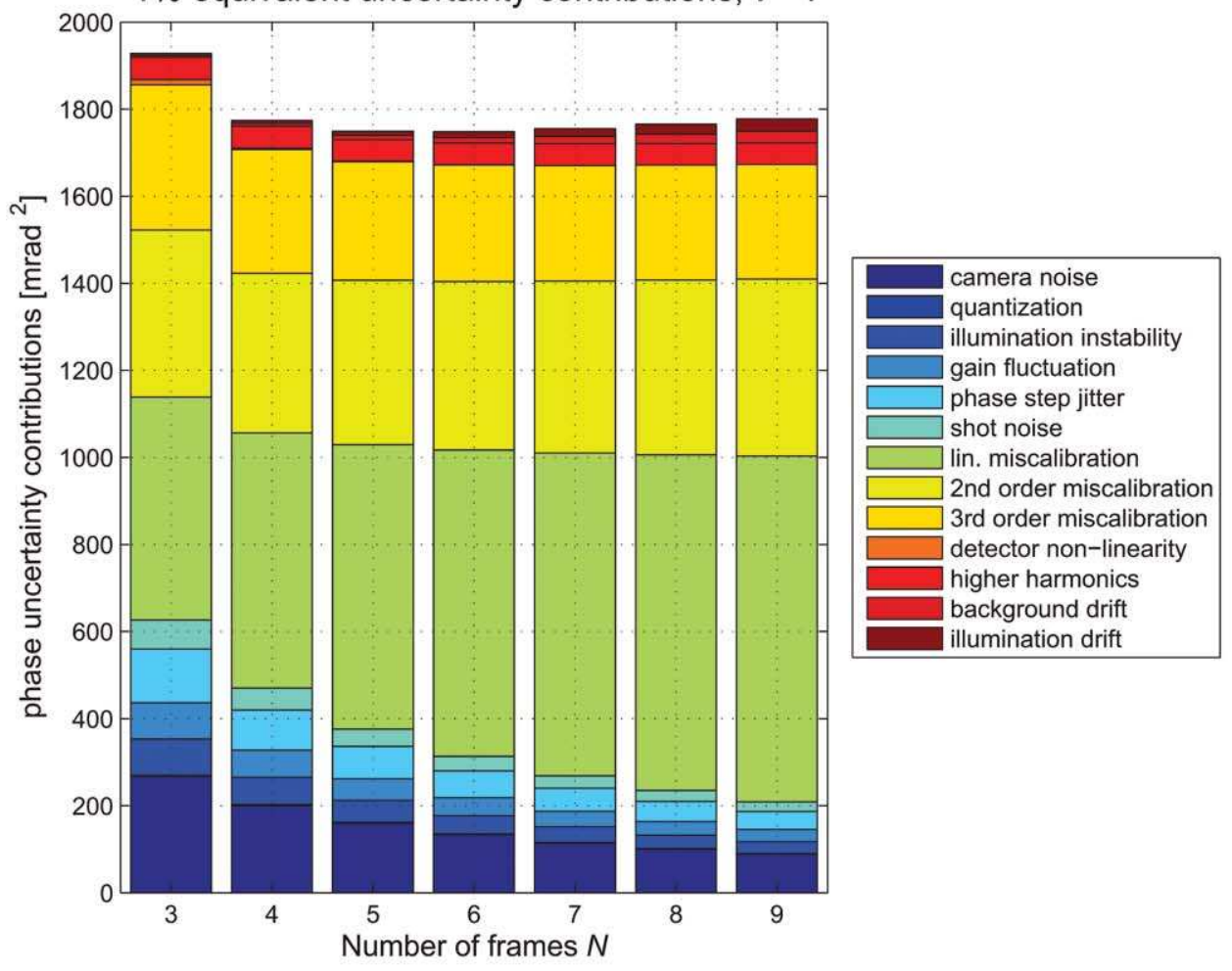

$1 \%$ equivalent uncertainty contributions, $v=0.25$

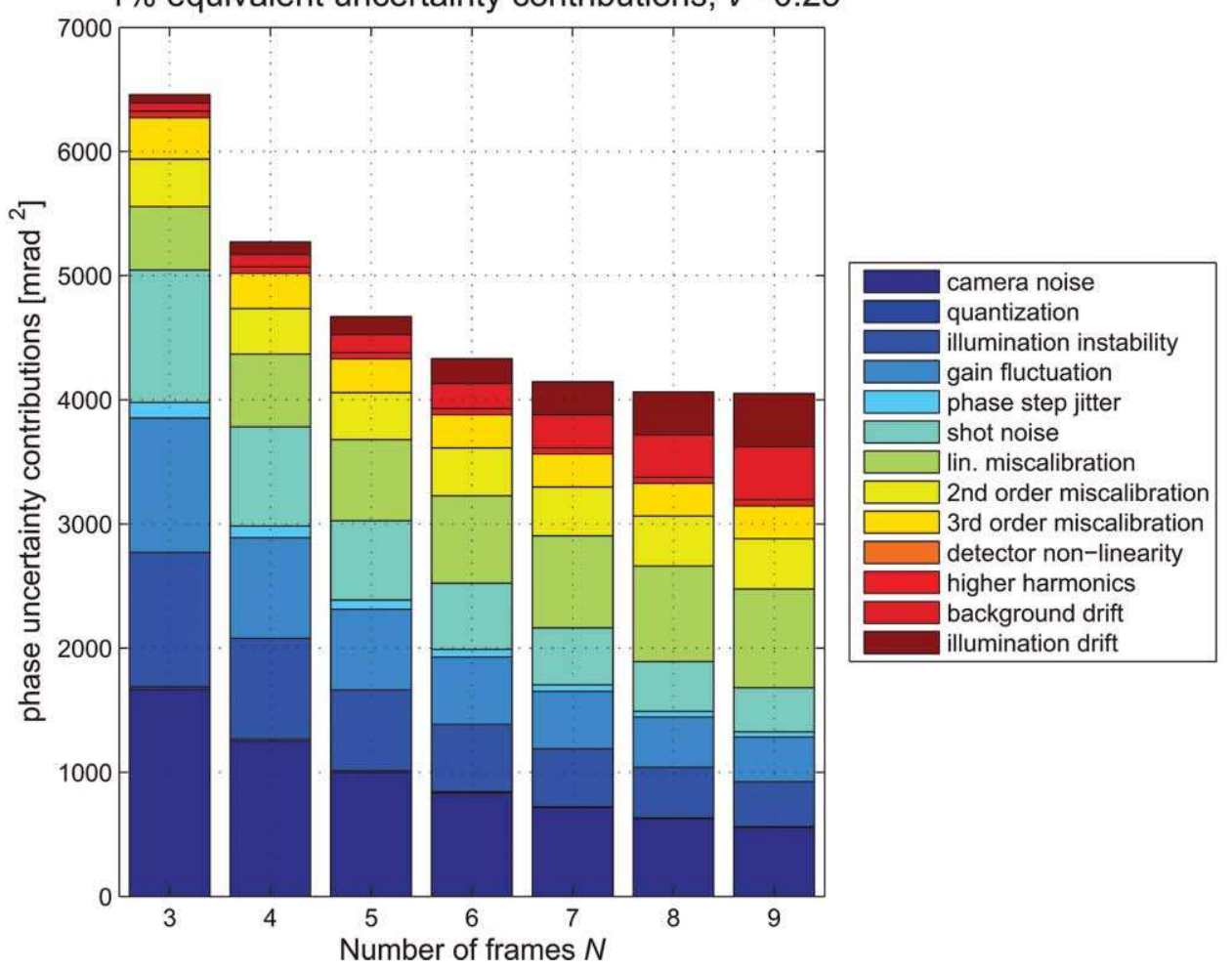

FIG. 8. (Color) Sum of squared phase uncertainties from $1 \%$ equivalent contributions for a visibility of $v=1$ (a) and $v=0.25$ (b). Values are for the average phase variance for the $N$-bucket algorithms, as given in Eq. (62), using an 8-bit camera. From Table I, the values used in the calculation are: $S_{\text {camnoise }}=0.01 \times 256 \mathrm{GL}$, $S_{\mathrm{LSB}}=1 \mathrm{GL}, r(I)=0.01, r(\gamma)=0.01, u(\beta)=0.01 \times \pi / 2, S_{B}=10000 \mathrm{cnts}, \varepsilon=0.01, e_{2}=0.01 \times 2 \pi / N /(N-1), e_{3}=0.01 \times 2 \pi / N /(N-1)^{2}, r_{N-1}=$ $0.01, S_{\Gamma N-1}=0.01 S_{\Gamma}, \delta_{\text {drift }}=0.01$ over 4 frames, and $\delta_{\text {illumdrift }}=0.01$ over 4 frames. 
TABLE II. Common phase step algorithms with their coefficients, F-value and angle values.

\begin{tabular}{|c|c|c|c|}
\hline \multirow[b]{2}{*}{ Algorithm (Reference) } & \multicolumn{3}{|l|}{$\frac{\mathbf{b}_{k}}{\mathbf{a}_{k}}$} \\
\hline & $F$ & Phase step and coefficient angle & Comments \\
\hline \multirow[t]{2}{*}{ Wyant 3 frame tilted T three-step (Ref. 26) } & $\frac{S_{0}-S_{1}}{S_{1}-S_{2}} \rightarrow \frac{(1-10)}{(01-1)}$ & $\beta_{k}=\left(k-\frac{1}{2}\right) \frac{\pi}{2}$ & \\
\hline & $F=0.500$ & $\delta_{k}=\left(\frac{2}{3}-k\right) \frac{3 \pi}{4}$ & $\begin{array}{c}\qquad \beta_{k}+\delta_{k}=(1-k) \frac{\pi}{4} \\
\text { Minimal requirement, no systematic error correction }\end{array}$ \\
\hline \multirow[t]{2}{*}{3 frame inverted T three-step (Refs. 26 and 47 ) } & $\frac{(10-1)}{(-12-1)}$ & $\beta_{k}=(k-1) \frac{\pi}{2}$ & $\mathbf{a} \cdot \mathbf{b}=0, \beta \neq \delta$ \\
\hline & $F=0.500$ & $\delta_{k}=(1-k) \frac{3 \pi}{4}$ & $\begin{array}{c}\qquad \beta_{k}+\delta_{k}=(1-k) \frac{\pi}{4} \\
\text { Minimal requirement, no systematic error correction }\end{array}$ \\
\hline 3 frame $120^{\circ}$ & 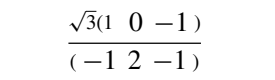 & $\beta_{k}=(k-1) \frac{2 \pi}{3}$ & $N$-bucket $(N=3)$ \\
\hline $120^{\circ}$ three-step (Refs. 26 and 47) & $F=0.333$ & $\begin{aligned} \beta_{k} & =\left(k+\frac{1}{2}\right) \frac{2 \pi}{3} \\
\delta_{k} & =(1-k) \frac{2 \pi}{3}\end{aligned}$ & $\begin{array}{l}\qquad \beta_{k}+\delta_{k}=0, \pi \\
\text { Minimal requirement, no systematic error correction, but } \\
\text { optimal for random errors }\end{array}$ \\
\hline \multirow[t]{2}{*}{4 steps in the cross (Ref. 26) } & $\frac{\left(\begin{array}{llll}0 & -1 & 0 & 1\end{array}\right)}{\left(\begin{array}{lllll}1 & 0 & -1 & 0\end{array}\right)}$ & $\beta_{k}=k \frac{\pi}{2}$ & \\
\hline & $F=0.250$ & $\delta_{k}=-k \frac{\pi}{2}$ & $\begin{array}{c}\qquad \beta_{k}+\delta_{k}=0 \\
\text { Insensitive to second harmonic, optimal for random errors }\end{array}$ \\
\hline 4 steps in $X$ (Ref. 26) & 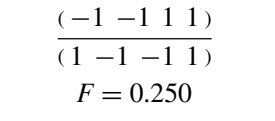 & $\begin{aligned} \beta_{k} & =\left(k+\frac{1}{2}\right) \frac{\pi}{2} \\
\delta_{k} & =\left(-\frac{1}{2}-k\right) \frac{\pi}{2}\end{aligned}$ & $\begin{array}{l}\qquad \beta_{k}+\delta_{k}=0 \\
\text { Insensitive to second harmonic, optimal for random errors }\end{array}$ \\
\hline$N$ frame $\pi / 2$ (Ref. 55) & 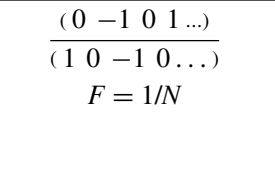 & $\begin{aligned} \beta_{k} & =k \frac{\pi}{2} \\
\delta_{k} & =-k \frac{\pi}{2}\end{aligned}$ & $\begin{array}{l}N \text { even } \\
\qquad \beta_{k}+\delta_{k}=0 \\
\text { Suppresses random errors, increases systematic errors, } \\
\text { especially from nonlinear phase step miscalibration }\end{array}$ \\
\hline Schwider-Hariharan 5-frame (Refs. 3 and 17) & 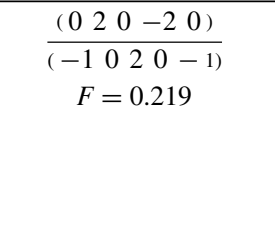 & $\begin{array}{l}\beta_{k}=(k-2) \frac{\pi}{2} \\
\delta_{k}=(2-k) \frac{\pi}{2}\end{array}$ & $\begin{array}{l}\qquad N+1 \text { bucket } \\
\qquad \beta_{k}+\delta_{k}=0 \\
\text { Insensitive to second harmonic and linear phase-step } \\
\text { miscalibration, also good against random noise; very } \\
\text { efficient formula }\end{array}$ \\
\hline Schmit and Creath 5-frame (Ref. 7) & 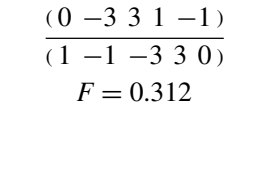 & $\beta_{k}=k \frac{\pi}{2}$ & $\begin{array}{l}\qquad \mathbf{a} \cdot \mathbf{b} \neq 0 \\
\text { No regular } \delta_{k} \\
\begin{array}{c}\text { Very good against linear phase-step miscalibration, reduced } \\
\text { noise rejection }\end{array}\end{array}$ \\
\hline Surrel 6-frame (Ref. 66) & 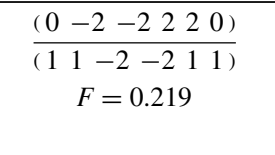 & $\beta_{k}=k \frac{\pi}{2}$ & $\begin{array}{l}\text { No regular } \delta_{k} \\
\text { Logical extension to Schwider-Hariharan formula; adds } \\
\text { phase-shift error tolerance at second harmonic }\end{array}$ \\
\hline deGroot 7-frame (Ref. 8) & 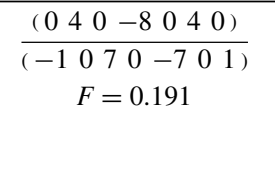 & $\begin{array}{l}\beta_{k}=(k-3) \frac{\pi}{2} \\
\delta_{k}=(2-k) \frac{\pi}{2}\end{array}$ & $\begin{array}{l}\qquad 4+3 \text { bucket } \\
\qquad \beta_{k}+\delta_{k}=-\frac{\pi}{2} \\
\text { Very tolerant of phase-shift errors; no tolerance to } \\
\text { phase-shift errors in second harmonic }\end{array}$ \\
\hline \multirow[t]{2}{*}{ Hibino 7-frame (Ref. 52) } & 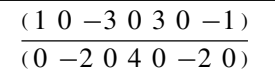 & $\beta_{k}=(k-3) \frac{\pi}{2}$ & $\beta_{k}+\delta_{k}=(k-1) \pi$ \\
\hline & $F=0.172$ & $\delta_{k}=(k+1) \frac{\pi}{2}$ & $\begin{array}{l}\text { Well-balanced logical extension to Surrel 6-sample } \\
\text { formula; adds irradiance drift tolerance, and suppresses } \\
\text { random noise efficiently; phase-shift range } 3 \pi\end{array}$ \\
\hline Larkin 7-frame (Ref. 18) & 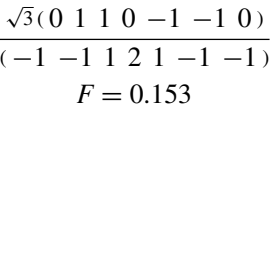 & $\begin{array}{c}\beta_{k}=k \frac{\pi}{3} \\
\delta_{k}=(3-k) \frac{\pi}{3}\end{array}$ & $\begin{array}{l}\qquad 6+1 \text { bucket } \\
\qquad \beta_{k}+\delta_{k}=\pi \\
60^{\circ} \text { formula; suppresses linear phase-step miscalibration } \\
\text { and all harmonics; also very good against random noise; } \\
\text { phase-shift range } 2 \pi \text {, better than Hibino formula under } \\
\text { phase-shift nonlinearity }\end{array}$ \\
\hline
\end{tabular}




$$
\begin{aligned}
\left\langle u_{N}^{2}(\varphi)\right\rangle_{\varphi}= & \frac{(\varepsilon \pi)^{2}}{N^{2}}\left\{(N-1)^{2}+\left(2 \sin ^{2}\left(\frac{2 \pi}{N}\right)\right)^{-1}\right\}+\delta_{\text {illum.drift }}^{2}\left(8 \sin ^{2}\left(\frac{2 \pi}{N}\right)\right)^{-1} \\
& +\frac{1}{2 N}\left\{\left(r^{2}(\gamma)+r^{2}(I)\right)+3 u^{2}(\beta)\right\} \\
& +\frac{1}{N^{2}} \sum_{p=2}^{P} e_{p}^{2}\left\{\left(\sum_{k=0}^{N-1} k^{p}\right)^{2}+\frac{1}{2}\left(\sum_{k=0}^{N-1} k^{p} \cos \left(k \frac{4 \pi}{N}\right)\right)^{2}+\frac{1}{2}\left(\sum_{k=0}^{N-1} k^{p} \sin \left(k \frac{4 \pi}{N}\right)\right)^{2}\right\} \\
& +\frac{2}{N v^{2}}\left\{\left(\frac{S_{\text {camnoise }}}{S_{B}}\right)^{2}+\frac{1}{12}\left(\frac{S_{\text {LSB }}}{S_{B}}\right)^{2}+\frac{1}{S_{B}[c t s]}+r^{2}(\gamma)+r^{2}(I)\right\} \\
& +\frac{\delta_{\text {drift }}^{2}+\delta_{\text {illum.drift }}^{2}+\frac{S_{N-1}^{2}}{2 v^{2} \sin ^{2}\left(\frac{\pi}{N}\right)}+\frac{1}{2}\left(\frac{v}{2}\right)^{2 N-4}\left(r_{N-1} S_{B}^{N-2}\right)^{2},}{}
\end{aligned}
$$

where we have from top to bottom the contributions due to (i) linear phase step miscalibration and illumination drift; (ii) gain and background fluctuations, phase step jitter; (iii) higher order miscalibrations; (iv) camera noise, digitization uncertainty, Poisson noise, gain, and background fluctuations; (v) background irradiance, illumination drift, and higher harmonics of order $N-1$; and detector nonlinearity. The sum of all quadratic contributions in Eq. (62) is illustrated in Fig. 8 for values of the uncertainty contributions of $1 \%$ of the respective quantity. This shows the relative importance of the contributions, their dependence on $N$ as well as the behaviour for two values of the visibility. Quantization noise as well as detector nonlinearity are hardly visible.

\section{CONCLUSIONS}

We have presented a systematic approach to the determination of measurement uncertainty for a general phase stepping technique based on the Arctan algorithm. For the important class of $\mathrm{N}$-bucket algorithms, we have presented analytical results that allow the user to estimate the individual uncertainty contributions of various influence quantities. Correlations have consistently been taken into account where an error source affects several input quantities. The variances of independent contributions have been added up. Many of the results that were reported in earlier papers have been reproduced with much less effort, and for several cases, a more general formula has been presented. The formulation in terms of scalar products and explicit sums over sine and cosine functions has turned out to be appropriate for the generalized treatment. The use of the CauchySchwarz inequality allowed us to derive upper bounds for the individual variances, which are proportional to the noise factor $F$. This allows for an easy comparison of different algorithms.

\section{APPENDIX: COMMON PHASE STEP ALGORITHMS OF THE ARCTAN TYPE}

In Table II column one identifies the algorithm; column two gives coefficients $a_{k}$ and $b_{k}$ of the algorithm $\tan \varphi=$ $(\mathbf{b} \cdot \mathbf{S}) /(\mathbf{a} \cdot \mathbf{S})$ in symbolic notation as well as their noise figure $F$, Eq. (27), while column three contains the nominal phase step angles $\beta_{k}$ and coefficient angles $\delta_{k}=\arctan \left(b_{k} / a_{k}\right)$. More comprehensive lists are found in Refs. 8 and 68.

\footnotetext{
${ }^{1}$ International Conference on Advanced Phase Measurement Methods in Optics and Imaging, Monte Verità, Ascona, Switzerland, 2010, edited by P. K. Rastogi and E. Hack, AIP Conference Proceedings 1236 (AIP, Melville, NY, 2010).

${ }^{2}$ J. H. Bruning, D. R. Herriott, J. E. Gallagher, D. P. Rosenfeld, A. D. White, and D. J. Brangaccio, Appl. Opt. 13, 2693 (1974).

${ }^{3}$ J. Schwider, R. Burow, K. E. Elssner, J. Grzanna, R. Spolaczyk, and K. Merkel, Appl. Opt. 22, 3421 (1983).

${ }^{4}$ K. Freischlad and C. L. Koliopoulos, J. Opt. Soc. Am. A 7, 542 (1990).

${ }^{5} \mathrm{M}$. Küchel, Method and apparatus for phase evaluation of pattern images used in optical measurement, U.S. Patent 5,361,312 (1 November 1994).

${ }^{6}$ J. Schwider, O. Falkenstörfer, H. Schreiber, A. Zöller, and N. Streibl, Opt. Eng. 32, 1883 (1993).

${ }^{7}$ J. Schmit and K. Creath, Appl. Opt. 34, 3610 (1995).

${ }^{8}$ P. de Groot, Appl. Opt. 34, 4723 (1995).

${ }^{9}$ H. Zhang, M. J. Lalor, and D. R. Burton. Opt. Eng. 38, 1524 (1999).

${ }^{10}$ Y. Surrel, Appl. Opt. 35, 51 (1996).

${ }^{11}$ D. W. Phillion, Appl. Opt. 36, 8098 (1997).

${ }^{12}$ P. Carré, Metrologia 2, 13 (1966).

${ }^{13}$ K. A. Goldberg and J. Bokor, Appl. Opt. 40, 2886 (2001).

${ }^{14}$ J. C. Estrada, M. Servín, and J. A. Quiroga, Opt. Express 18, 2632 (2010).

${ }^{15}$ A. Patil, P. Rastogi, and R. Langoju, Opt. Lett. 30, 391 (2005).

${ }^{16}$ R. Langoju, Phase estimation methods and their application to holographic interferometry, Thesis EPFL No. 3625, Lausanne, 2006, see http://biblion.epfl.ch/EPFL/theses/2006/intranet/EPFL_TH3625.pdf.

${ }^{17}$ P. Hariharan, B. F. Oreb, and T. Eiju, Appl. Opt. 26, 2504 (1987).

${ }^{18}$ K. G. Larkin and B. F. Oreb, J. Opt. Soc. Am. A 9, 1740 (1992).

${ }^{19}$ Y. Surrel, Appl. Opt. 32, 3598 (1993).

${ }^{20}$ K. Hibino, Appl. Opt. 36, 2084 (1997).

${ }^{21}$ M. Servín, D. Malacara, J. Marroquin, and F. Cuevas, J. Mod. Opt. 44, 1269 (1997).

${ }^{22}$ J. Schwider, T. Dresel, and B. Manzke, Appl. Opt. 38, 655 (1999).

${ }^{23}$ M. Chen, H. Guo, and C. Wei, Appl. Opt. 39, 3894 (2000).

${ }^{24}$ J. Hernández López and D. Malacara Hernández, Opt. Commun. 180, 9 (2000).
} 
${ }^{25}$ Y. Zhu and T. Gemma, Appl. Opt. 40, 4540 (2001).

${ }^{26} \mathrm{D}$. Malacara, M. Servín, and Z. Malacara, Interferogram Analysis for Optical Testing, 2nd ed. (Taylor \& Francis, Boca Raton, 2005).

${ }^{27}$ J. Novák, P. Novák, and A. Mikš, Opt. Commun. 281, 5302 (2008).

${ }^{28}$ J. C. Estrada, M. Servin, and J. A. Quiroga, Opt. Lett. 34, 413 (2009).

${ }^{29} \mathrm{C}$. Ai and J. C. Wyant, Appl. Opt. 26, 1112 (1987).

${ }^{30}$ J. Schmit and K. Creath, Appl. Opt. 35, 5642 (1996).

${ }^{31}$ H. Zhang, M. J. Lalor, and D. R. Burton, Opt. Lasers Eng. 31, 381 (1999).

${ }^{32}$ J. Schwider, Appl. Opt. 28, 3889 (1989).

${ }^{33}$ X. Chen, M. Gramaglia, and J. A. Yeazell, Appl. Opt. 39, 585 (2000).

${ }^{34}$ C. S. Guo, L. Zhang, H. T. Wang, J. Liao, and Y. Y. Zhu, Opt. Lett. 27, 1687 (2002).

${ }^{35}$ Z. Wang and B. Han, Opt. Lett. 29, 1671 (2004).

${ }^{36}$ L. Z. Cai, Q. Liu, Y. R. Wang, X. F. Meng, and M. Z. He, Appl. Opt. 45, 1193 (2006).

${ }^{37}$ R. Langoju, A. Patil, and P. Rastogi, Opt. Lett. 31, 1058 (2006).

${ }^{38}$ K. Okada, A. Sato, and J. Tsujiuchi, Opt. Commun. 84, 118 (1991).

${ }^{39}$ G. Stoilov and T. Dragostinov, Opt. Lasers Eng. 28, 61 (1997).

${ }^{40}$ C. Wei, M. Chen, and Z. Wang, Opt. Eng. 38, 1357 (1999).

${ }^{41}$ P. Picart, Opt. Lasers Eng. 35, 335 (2001).

${ }^{42}$ R. Schödel, A. Nicolaus, and G. Bönsch, Appl. Opt. 41, 55 (2002).

${ }^{43}$ L. Yongqian, Z. Zhenyu, and L. Xiaoying, Meas. Sci. Technol. 16, 1335 (2005).

${ }^{44}$ H. Guo, Y. Yu, and M. Chen, J. Opt. Soc. Am. A 24, 25 (2007).

${ }^{45} \mathrm{~J}$. Schwider, Advanced Evaluation Techniques in Interferometry, inProgress in Optics XXVIII, edited by E. Wolf (Elsevier, New York, 1990), pp.271-359.

${ }^{46}$ R. Thalmann and R. Dändliker, Principles and Limitations of PhaseShifting Techniques in Holographic Interferometry, in Application of Metrological Laser Methods in Machines and Systems, edited by G. Frankowski (Akademie, Berlin, 1991).

${ }^{47}$ H. Schreiber and J. H. Bruning, Phase Shifting Interferometry, in Optical Shop Testing, edited by D. Malacara, 3rded. (Wiley, New York, 2007), pp. 547-666.

${ }^{48}$ K. Creath and J. Schmit, Opt. Lasers Eng. 24, 365 (1996).

${ }^{49}$ J. M. Huntley, Automated Analysis of Speckle Interferograms, in Digital Speckle Pattern Interferometry and Related Techniques, edited by P. K. Rastogi (Wiley, New York, 2001).

${ }^{50}$ K. J. Gåsvik, Optical Metrology, 3rd ed. (Wiley, New York, 2002).

${ }^{51}$ K. Creath, Surface Characterization and Testing, Proceedings of SPIE 0680, edited by K. Creath (Bellingham, Washington, USA, 1986), p. 19

${ }^{52}$ K. Hibino, B. F. Oreb, D. I. Farrant, and K. G. Larkin, J. Opt. Soc. Am. A 12, 761 (1995).

${ }^{53}$ R. R. Cordero, J. Molimard, A. Martinez, and F. Labbe, Opt. Commun. 275, 144 (2007).

${ }^{54}$ X. F. Xu, L. Z. Cai, Y. R. Wang, X. F. Meng, H. Zhang, X. C. Cheng, G. Y. Dong, and X. X. Shen, J. Mod. Opt. 55, 1291 (2008).
${ }^{55}$ C. P. Brophy, J. Opt. Soc. Am. A 7, 537 (1990).

${ }^{56}$ J. H. van Wingerden, H. J. Frankena, and C. Smorenburg, Appl. Opt. 30, 2718 (1991).

${ }^{57}$ J. F. Mosiño, M. Servín, J. C. Estrada, and J. A. Quiroga, Opt. Express 17, 5618 (2009).

${ }^{58}$ Guide to the Uncertainty in Measurement (GUM), ISO/IEC Guide 983:2008 (ISO, Geneva, 2008).

${ }^{59}$ R. R. Cordero, F. Labbe, A. Martinez, and R. Rodríguez-Vera, Opt. Commun. 252, 229 (2005).

${ }^{60}$ J. E. Greivenkamp, Opt. Eng. (Bellingham) 23, 350 (1984).

${ }^{61}$ G. Lai and T. Yatagai, J. Opt. Soc. Am. A 8, 822 (1991).

${ }^{62}$ H. Ryu and C. Hong, J. Opt. Soc. Am. A 14, 1051 (1997).

${ }^{63}$ E. W. Rogala and H. H. Barrett, Appl. Opt. 37, 7253 (1998).

${ }^{64}$ M. Milman and S. Basinger, Appl. Opt. 41, 2655 (2002).

${ }^{65}$ N. Ohyama, S. Kinoshita, A. Cornejo-Rodriguez, T. Honda, and J. Tsujiuchi, J. Opt. Soc. Am. A 5, 2019 (1988).

${ }^{66}$ B. Zhao and Y. Surrel, Opt. Eng. 34, 2821 (1995).

${ }^{67}$ R. N. Bracewell, The Fourier Transform \& its Applications, 2nd ed. (McGraw-Hill, New York, 1975).

${ }^{68}$ Y. Surrel, Appl. Opt. 36, 271 (1997).

${ }^{69}$ C. Rathjen, J. Opt. Soc. Am. A 12, 1997 (1995).

${ }^{70}$ Y. Surrel, Customized Phase Shift Algorithms, in Trends in Optical Nondestructive Testing and Inspection, edited by P. K. Rastogi and D. Inaudi (Elsevier, Amsterdam, 2000).

${ }^{71}$ H. Helmers and M. Schellenberg, Opt. Laser Technol. 35, 587 (2003).

${ }^{72}$ B. Zhao and Y. Surrel, Appl. Opt. 36, 2070 (1997).

${ }^{73}$ E. Hack, ESPI - Principles and Prospects, in Trends in Optical Nondestructive Testing and Inspection, edited by P. K. Rastogi and D. Inaudi (Elsevier, Amsterdam, 2000), pp. 207-222.

${ }^{74}$ O. A. Skydan, F. Lilley, M. J. Lalor, and D. R. Burton, Appl. Opt. 42, 5302 (2003).

${ }^{75} \mathrm{R}$. Loudon, The Quantum Theory of Light, 3rd ed. (Oxford University Press, New York, 2000).

${ }^{76}$ W. Yashiro, Y. Takeda, and A. Momose, J. Opt. Soc. Am. A 25, 2025 (2008).

${ }^{77}$ D. Malacara Doblado and B. V. Dorrío, J. Opt. Soc. Am. A 17, 1857 (2000).

${ }^{78}$ H. Bi, Y. Zhang, K. V. Ling, and C. Wen, Appl. Opt. 43, 4199 (2004).

${ }^{79}$ Y. Surrel, J. Opt. Soc. Am. A 15, 1227 (1998).

${ }^{80}$ K. A. Stetson and W. R. Brohinsky, Appl. Opt. 24, 3631 (1985).

${ }^{81}$ N. Ohyama, T. Shimano, J. Tsujiuchi, and T. Honda, Opt. Commun. 58, 223 (1986).

${ }^{82}$ K. Kinnstaetter, A. W. Lohmann, J. Schwider, and N. Streibl, Appl. Opt. 27, 5082 (1988).

${ }^{83}$ C. Han and B. Han, Appl. Opt. 45, 1124 (2006).

${ }^{84}$ R. Onodera and Y. Ishii, J. Opt. Soc. Am. A 13, 139 (1996).

${ }^{85}$ Y. Surrel, Appl. Opt. 36, 805 (1997). 